\title{
Anti-inflammatory Effect of Active Nanofibrous Polymeric Wound Dressing Bearing Polysaccharide Nanocontainers of Atorvastatin Complexes
}

Pascale Schwinté*, Alexandre Mariotte, Priya Anand, Olivier Huck, Ysia Idoux-Gillet, Florence Fioretti, Henri Tenenbaum, Philippe Georgel, Wolfgang Wenzel, Silvia Irusta, Nadia BenkiraneJessel*

Dr. P. Schwinté*, Pr. O. Huck, Dr. Y Idoux-Gillet, Dr. F. Fioretti, Pr. H. Tenenbaum, and Dr. N. Jessel*,

INSERM (French National Institute of Health and Medical Research), UMR 1109, “Osteoarticular and Dental Regenerative Nanomedicine”, Faculté de Médecine, 11 rue Humann, FMTS, Strasbourg, F-67085, France and Université de Strasbourg, Faculté de Chirurgie Dentaire, 1 place de l'Hôpital, Strasbourg F-67085, France

Nadia.jessel@inserm.fr

Pschwinte@unistra.fr

A. Mariotte, and Pr. P. Georgel, 
INSERM UMR 1109, Molecular ImmunoRheumatology, LabEx TRANSPLANTEX, Centre de Recherche d'Immunologie et d'Hématologie, Faculté de Médecine, 4 rue Kirschleger, FMTS, Université de Strasbourg, Strasbourg,

F-67085, France

Dr. S. Irusta,

Department of Chemical Engineering and Aragon Nanoscience Institute, University of Zaragoza, C/Mariano Esquillor, s/n, Zaragoza,

50018, Spain

Dr. P. Anand, and Pr. W. Wenzel,

Institute of Nanotechnology, Karlsruhe Institute of Technology, KIT Campus North, Hermannvon-Helmholtz-Platz 1, Building 640, Eggenstein-Leopoldshafen,

D-76344, Germany

Keywords: inflammation, atorvastatin, cyclodextrin, nanocontainers, polymeric wound dressing 
Modulation of inflammation is a key point in healing processes. Statins, well known for their use in lowering cholesterol levels, have potential therapeutic effects in wound healing and bone regeneration, as anti-inflammatory effectors. In order to increase treatment efficiency in specific applications such as bone/cartilage lesions, periodontal lesions, rheumatic joint lesions, we develop a polymeric wound dressing for local and sustained anti-inflammatory administration. This wound dressing is based on a nanofibrous electrospun polycaprolactone membrane, containing polysaccharide nanoreservoirs of atorvastatin, in the form of inclusion complexes with cyclodextrin. The physicochemical properties of this system are studied in detail, and its inhibitory effect on inflammatory cytokine production (TNF- $\alpha$, IL-6) by human macrophages over time is demonstrated. The innovative aspect of this work is the combination of nanotechnologies as versatile delivery system, exploiting the properties of cyclodextrins 
as drug vehicles and their further embedding in local nanocontainers. The strategy can be used with other therapeutic agents, or combination of agents to target other diseases.

\section{Introduction}

Modulation of inflammatory host-response: Inflammation represents the normal response to infection. However, when getting out of control, inflammation may lead to autoimmune or autoinflammatory disorders, neurodegenerative disease, and even cancer. Many effective anti-inflammatory drugs are available (rev Dinarello 2010), including aspirin and other nonsteroidal antiinflammatory drugs (NSAIDs). World famous aspirin (acetylated salicylic acid) developed by Felix Hoffman inhibits the cyclooxygenase (COX) enzymes COX-1 and COX-2, which synthesize inflammatory mediators: prostaglandins and thromboxanes. Nonsteroidal anti-inflammatory drugs (NSAIDS) target COX-2 and the synthesis of prostaglandins, in particular PGE2. Glucocorticoids, derived from natural cortisol, are also widely used as antiinflammatories, despite their side effects. Inflammation is a biological process involving proinflammatory cytokines such as tumor necrosis factor (TNF)- $\alpha$, interleukin (IL)-1 $\beta$, and vascular endothelial growth factor (VEGF). Thus, new varieties of anti-inflammatory agents include biological anticytokine molecules (anticytokine therapies) that reduce the activity of specific cytokines or their receptors. Anti-cytokine therapies have shown their benefits in the treatment of autoimmune diseases, such as rheumatoid arthritis, multiple sclerosis, inflammatory bowel disease, etc. However their major drawback is a decreased host immune defence against infection. Compared to the side effects of glucocorticoid treatment on the long-term, anti-cytokine therapies constitute a big improvement, as they do not induce organ toxicities while their action is located in extracellular compartments. Kinases that act downstream of cytokine receptors have become new targets in the control of inflammation, 
and molecules blocking the activity of kinases are being developed. Among other new drugs with anti-inflammatory properties, active inhibitors of histone deacetylases were also discovered, which block cell proliferation, as well as naturally occuring resolvins (rev Dinarello 2010). Statins, a family of drugs used for lowering serum cholesterol, also have antiinflammatory properties. Statins (or 3-Hydroxy-3-methyl-gutaryl coenzyme A (HMG-CoA) reductase inhibitors) are primarily used for the treatment of hypercholesterolemia: they are the most efficient drugs to reduce serum cholesterol levels (Arnaud, et al., 2005). Statins possess diverse pleiotropic effects such as anti-inflammatory, anti-oxidative, antibacterial and immune-modulatory effects (Blanco-Colio 2003, Arnaud 2005). Today, there are several studies about statins for their potential therapeutic effects in wound healing and bone regeneration (Farsaei, et al., 2012; Kinra \& Khan, 2011). Other studies suggest that statins may be helpful for reducing bacterial burden, improving epithelisation and wound healing (Farsaei, et al., 2012). Anti-thrombotic and anti-inflammatory functions have been recently reported for this class of molecules (Ciurleo et al., 2014). Indeed, simvastatin has been shown to modulate LPS-dependant cytokine production in vitro and in vivo (Niessner et al., 2006), although the precise mechanism underlying such immunomodulatory functions is not yet precisely known (Wittebole et al., 2010).

Following randomized, placebo-controlled studies, the anti-inflammatory properties of statins vary in function of the statin and the dose, whereas the cholesterol-lowering properties are similar. For example, six clinically used statins were compared in vitro, and cerivastatin, atorvastatin, and simvastatin were more effective in reducing the studied inflammation parameters than fluvastatin, lovastatin, or pravastatin (Hilgendorff et al., 2003).

In the example of rheumatoid arthritis, there have been several trials of statins resulting in a moderate reduction in joint inflammation. As many patients suffering from this disease are also at risk for cardiovascular disease, adding statin therapy to the standard drug, 
methotrexate, is highly beneficial. Similarly, the addition of statins to cyclosporine for kidney transplant patients reduced the percentage of organ rejection. In patients suffering from multiple sclerosis, 6 months statin therapy significantly reduced the number of brain lesions detected. Examination of peripheral blood cells revealed an increase in production of IL-10, an anti-inflammatory cytokine (Paul et al., 2008).

Several host modulation therapies have been developed to regulate immune and inflammatory response, through a systemic administration or a local administration (with the use of membranes), in order to enhance wound healing. The aim is to restore a balance between pro-inflammatory mediators (destructive enzymes) and anti-inflammatory mediators (enzyme inhibitors). These molecules can be antibiotic drugs (azithromycin, minocycline, doxycycline and tetracycline) (Hirsch, 2010 ) (Minabe, et al., 2001), anti-inflammatory drugs (statin, ibuprofen) (Chen, et al., 2010) (Sakoda, et al., 2006) (Usui, et al., 2008) and resolving (Thomas \& Puleo, 2011).

Properties of scaffold matrices: The design of a scaffold for tissue engineering purposes has to take into account some requirements like chemical properties, morphology and kinetics of degradation in order to obtain a three-dimensional architecture, with designed characteristics. Moreover, the scaffold should have a high porous network to facilitate cell attachment, proliferation, migration, and differentiation, and to allow transport of nutrients and metabolic waste. It should also be biocompatible and biodegradable. However, its degradation has to be precisely controlled during time to accompany regenerated neo-tissues along their stage of growth, remodeling and maturation (Alsberg, et al., 2003).

The scaffold will be used as a delivery system for encapsulation of active biomolecules and their release in a sustained and controlled manner. Studies have shown that different types of delivery methods can be adapted to specific characteristics of wound sites. The challenge is to optimise the timing of therapeutic molecules expression on the wound site, while minimising 
the risks for patients (Chen, et al., 2009). In order to incorporate active biomolecules, the Layer-by-Layer technology has been developed. This method is based on the alternate deposition of polyanions and polycations (Decherxxx; Richert, et al., 2004) (Ladam, et al., 2000), and allows to create a functionalised film with specific molecules, proteins or particles. A specific application of this principle has been developed by Jessel et al (Mendoza 2012) in the form of local nanoreservoirs of adsorbed therapeutics distributed along the fibers of nanofibrous scaffolds, and acting as cell-contact-dependent source of local therapeutics with sustained availability.

However, some drugs may not be very charged or not water-soluble, and consequently will not attach to the opposite polyelectrolyte. To counteract this issue, the use of cyclodextrins (CDs) can be very helpful. Cyclodextrins are cyclic oligosaccharides made of several $\alpha-(1,4)$ linked Dglucose units. Typically, $\beta$-cyclodextrin is composed of 7 glucose units and presents a hollow cone shape, with a lipophilic cavity and a hydrophilic outer surface. Cyclodextrins have the ability to form inclusion complex with various guest molecules, by taking up some non-polar part of the guest molecule into its hydrophobic cavity (Salústio, et al., 2009).Thereby, cyclodextrins have been reported to improve physicochemical properties of hydrophobic drugs, in particular their water solubility, dissolution rate, and stability (Loftsson 1996, Frömming-Szejtli 1994; Loftsson, et al., 2002). Cyclodextrins can be substituted on both their primary or secondary side hydroxyls. For instance, many studies have reported the increased solubility of hydroxypropyl- $\beta$-cyclodextrin, its greater complexing properties and biocompatibility (Gould 2005).

The specific strategy of using substituted CDs to incorporate sparingly soluble drugs into layerby-layer bioactive coatings, has been successfully applied to anti-inflammatory polypeptide multilayer coatings embedding piroxicam NSAID, as a complex with a $6^{A}$-carboxymethylthio- $\beta$ cyclodextrin) (ref BenkiranePX). 
The first objective of the present study was to find a way to incorporate an anti-inflammatory statin drug (Atorvastatin calcium trihydrate, or $\mathrm{AT}$ ) into a poly ( $\varepsilon$-caprolactone) (PCL) nanofibrous membrane, with a view to produce an effective biocompatible anti-inflammatory wound patch, which could be used in various applications such as periodontal or rheumatic diseases for instance. We describe here a functionalization of the nanofibrous scaffold with the nanoreservoir technology to achieve effective embedding of atorvastatin calcium trihydrate (Figure $1 \mathrm{~A}$ ) into nanoreservoirs, through encapsulation by an amino- $\beta$-cyclodextrin polymer (Figure 1B). Along with this study, we realised physico-chemical studies on atorvastatin and its complex. The second objective was to investigate the impact of PCL membranes loaded with the anti-inflammatory statin molecule on cytokine expression. As a model system, atorvastatin-containing PCL membranes were seeded with control or LPS-stimulated THP-1 cells and we monitored TNF- $\alpha$ and IL-6 expression.

\section{Results and Discussion}

\subsection{Suggestion of Inclusion Complexation by Fluorescence Behaviour}

Fluorescence properties of atorvastatin (constant concentration of $1 \mu \mathrm{gL}^{-1}$ ) in the absence and presence of increasing quantities of amino- $\beta-\mathrm{CD}$ polymer in MES pH 5.5 (concentration ranging from $93 \mu \mathrm{g} \mathrm{mL}^{-1}$ to $3.75 \mathrm{mg} \mathrm{mL}^{-1}$ ) were examined as a means of evaluation of the drug microenvironment.

Atorvastatin presents amphiphilic characteristics, which can lead to micellar aggregation properties. It has a hydrophilic tail (dihydroxyheptanoic acid) associated to a large hydrophobic moiety composed of mainly 3 aromatic substituents (phenyl, fluorophenyl, phenylcarbamoyl) as well as a propanyl-pyrrole. The phenylcarbamoyl substituent is actually the combination of a phenyl group linked to an amide bond. The aromatic substituents can be involved in 
hydrophobic interactions such as $\pi$-stacking and intercalation. First, atorvastatin reveals by its emission spectrum a differing environment when increasing its concentration (Figure 2A): at $0.1 \mu \mathrm{g} \mathrm{mL}^{-1}$ there is a peak at about $330 \mathrm{~nm}$; at $1 \mu \mathrm{g} \mathrm{mL} L^{-1}$ this peak is supplemented by another shoulder at $380 \mathrm{~nm}$; at $2 \mu \mathrm{g} \mathrm{mL}^{-1}$ only the red shifted peak at $380 \mathrm{~nm}$ is present, also at $20 \mu \mathrm{g}$ $\mathrm{mL}^{-1}$ along with a markedly increase in fluorescence intensity (5-fold). This red shift in emission wavelength together with the significant increase in fluorescence intensity suggests enhanced aromatic interactions, such as face to face $\pi-\pi$ stacking of the aromatic groups (ref Xie).

At higher concentration in MES (saturated $20 \mu \mathrm{g} \mathrm{mL}^{-1}$, filtered), the spectral characteristics of the drug could actually be explained by the formation of micellar aggregates which could arise from $\pi$ stacking for instance, leading to a hydrophobic core from the three aromatic substituents and an outer hydrophilic surface made of the drug carboxy chain (ref xie). We have observed such micellar assemblies (see SEM observations). Furthermore, aggregation properties of atorvastatin were suggested also by the deviation from the Beer-Lambert law, in the UV-vis absorbance calibration curves realised both in MES-MeOH and PBS-MeOH, where the response of the solute is linear in dilute concentration range, up to respectively 0.25 and $0.4 \mathrm{mg} \mathrm{mL}^{-1}$, but displays a parabolic plateau tendency at higher concentration, which can be explained by diffusion phenomena (not shown).

As seen on Figure $2 B$, the presence of amino- $\beta-C D$ polymer at a concentration above $750 \mu \mathrm{g}$ $\mathrm{mL}^{-1}$ considerably increases the fluorescence intensity of the drug (16 fold, for the highest CD concentration studied). The increase in fluorescence intensity means that the drug encounters a more apolar environment. This could be due either to encapsulation of the drug in the cyclodextrin cavities on the polymer, and/or formation of nanoplexes (drug-polyelectrolyte nanoparticle complex) based on electrostatic and hydrogen bonds interactions between drug micellar aggregates (with outer hydrophilic carboxylic substituents) and polymer chains (with CD amino groups) (Sin Cheow 2012, 2012bis). The increase in the fluorescence intensity of 
similar hydrophobic drugs upon addition of $C D$ was often used as a demonstration of the inclusion of the drug in the CD cavity, as the hydrophobic micro-environment provided by this inclusion boosts the fluorescence yield of the drug (Refs BenkiranePX, Yoon). Interestingly, the fluorescence peak of atorvastatin at $381 \mathrm{~nm}$ is red-shifted to $400 \mathrm{~nm}$ in the presence of CD polymer. This is quite unusual, as CD strong binding of aromatic drugs generally produces a blue shift in wavelength. A similar red shift had been encountered in the case of piroxicam complexation by a carboxy CD (ref BenkiranePX) and was explained by Yoon et al by the existence of different prototropic forms of the drug, leading to an excited state intramolecular proton transfer (ESIPT) (hydroxyl to carbonyl), favored by the existence of intramolecular hydrogen bonds in apolar environment (ref Yoon).

Finally, an extrapolation of the apparent binding constant of the "1:1" complex can be obtained from the double reciprocal plot (Benesi-Hildebrand plot) $1 / \Delta \mathrm{l}$ as a function of $1 /$ concentration(ligand) where $\Delta \mathrm{l}$ is the relative fluorescence intensity (Figure $2 \mathrm{C}$ ). A $1: 1$ complexation is suggested by the linear portion of this plot. As described in details by Connors (ref Connors) with appropriate approximations, such as the fact that the ligand (here CD polymer) does not fluoresce, the resulting fluorescence equation of the system is given by $1 / \Delta l$ $=\mathbf{i}+\left(\mathbf{i} / \mathbf{K}_{11}\right) \cdot\left(\mathbf{1} / \mathrm{C}_{\mathrm{L}}\right)$, where $\mathbf{i}$ is the intercept, $\boldsymbol{K}_{11}$ the binding constant of the complex, and $C_{L}$ the concentration of ligand ( $C D$ polymer), and an estimation of the binding constant is given by the intercept to slope ratio. The Benesi-Hildebrand plot leads to an apparent $K_{11}$ value of $2850 \mathrm{M}^{-1}$ when using $C D$ polymer concentration, and $142 \mathrm{M}^{-1}$ when using the concentration of cyclodextrin units corresponding to this polymer concentration (linear regression, $R^{2}=0.98871$ ). This $C D$ unit concentration has been estimated knowing the polymer average molecular weight (25000 $\left.\mathrm{g} \mathrm{mol}^{-1}\right)$, the average CD content of the polymer $(70 \%$, given by the product information sheet of the supplier) and the amino-CD unit molecular weight $\left(1170.5 \mathrm{~g} \mathrm{~mol}^{-1}\right)$. This gives an average of $15 \mathrm{CD}$ units on the polymer chain. The stability of the atorvastatin/amino- $\beta-C D$ polymer complex as estimated this way is comparable to values 
obtained by phase solubilty studies of the atorvastatin complex with $\beta-C D\left(248 \mathrm{M}^{-1}\right.$, by Shivanand) or HP- $\beta-C D$ (312 $\mathrm{M}^{-1}$ by Shivanand; $164 \mathrm{M}^{-1}$ by Lv) (refs shivanand; Lv)

\subsection{Solubilisation of Atorvastatin by Amino- $\beta-C D$ Polymer}

The results of atorvastatin solubilisation experiments realised with increasing concentrations of amino- $\beta-C D$ polymer in MES are displayed in Figure 2D, expressed as the concentration (in $\mathrm{mol}^{-1}$ ) of atorvastatin solubilised in MES, as a function of the amino- $\beta$-CD polymer concentration (in mol I ${ }^{-1}$ ). The absorbance results were all sitting in the linear portion of the calibration curve. In the range of polymer concentrations studied (limited by its own solubility), the solubility diagram is linear. As explained by K. A. Connors (ref Connors) the basic principle is that the increased solubility of the substrate (the drug) is due to the formation of one or several complexes $S_{m} L_{n}$ by interaction of the substrate with the ligand (the $C D$ polymer). $A$ linear diagram generally means that all the complexes are first-order with respect to the ligand $(n=1)$. When the slope of a linear solubility diagram is greater than unity, at least one complex is present for which $m>1$, however if the slope is less than unity, it does not necessarily mean that only a 1:1 complex is formed. The apparent binding constant can be derived from the linear solubility diagram. Thus, if $s$ is the slope and $\boldsymbol{i}$ the intercept, the stability constant for the 1:1 complex is given by $\mathbf{K}_{\mathbf{1 1}}=\mathbf{s} / \mathbf{i}(\mathbf{1}-\mathbf{s})$ (ref Connors, p271). From the plot linear regression parameters (slope, intercept), we get an apparent binding constant of $2444 \mathrm{M}^{-1}$, with respect to polymer molar concentration, and $368 \mathrm{M}^{-1}$ with respect to $\mathrm{CD}$ unit molar concentration, which is reasonably strong for a hydrophobic drug/ $\beta-C D$ inclusion complex. It is very similar in magnitude, though higher, as $\boldsymbol{K}_{11}$ values obtained from similar solubility diagrams, reported by Shivanand et al. (ref shivanand) for the $\beta-\mathrm{CD}$ complex in water at $\mathrm{pH} 6.8$ and $25^{\circ} \mathrm{C}\left(248 \mathrm{M}^{-1}\right)$ and for the hydroxypropyl- $\beta-C D\left(312 \mathrm{M}^{-1}\right)$, and also by Hussein et al (ref hussein) for the $\beta-\mathrm{CD}$ complex in water $\left(252 \mathrm{M}^{-1}\right)$ and for the hydroxypropyl- $\beta-C D\left(219 \mathrm{M}^{-1}\right)$; Lv et al reported a quite lower value at $25^{\circ} \mathrm{C}$ in water for hydroxypropyl- $\beta-\mathrm{CD}\left(164 \mathrm{M}^{-1}\right)($ ref Lv), as did Taksande et al 
(ref taksande) for $\beta-C D\left(53 \mathrm{M}^{-1}\right)$ and hydroxypropyl- $\beta-\mathrm{CD}\left(120 \mathrm{M}^{-1}\right)$. The stability we observed is though surprisingly lower than what we reported earlier for the complexation of some hydrophobic aromatic anti-inflammatory NSAID molecule, piroxicam, with a charged $6^{A}$ carboxy-methylthio- $\beta$-cylodextrin, used for the build-up of anti-inflammatory multilayered coatings (ref benkiranePx). In this case, the high stability could be explained by a cooperative effect between hydrophobic inclusion in the CDs cavities and supplementary electrostatic and/or hydrogen bonding interactions between the $C D$ amino groups and the carboxy chains of the drug, as reported earlier (ref schwinté JC S perkin trans2, 1998, 805). We could have expected some similar cooperative effect in the present case, through strong interaction between the carboxylic chain of atorvastatin and the CD amino group, at the CD lower rim, supplementing the aromatic inclusion of the pyridyl engulfed in the cavity, however this is probably hindered by the shorter size (lack of linker) of the amino substituent which does not enable a deep engulfing of both the long carboxylic chain and the aromatic core of the drug. To have an insight into the molecular complexation features of our system, we have performed molecular dynamics simulations.

\subsection{Atorvastatin/Amino- $\beta$-Cyclodextrin Complex Simulations}

We investigated potential complexes of atorvastatin (AT) with amino- $\beta$-cyclodextrin (CD). AT is an aromatic heterocyclic compound with a structure based on a core pyrrole ring linked on one side to a 3,5-dihydroxy heptanoic acid chain, and a propan-2-yl group, and on the other side to a fluorophenyl group, a phenyl group and a phenylcarbamoyl group (Figure 1A). The interaction with amino- $\beta-C D$ would increase the solubility of AT by binding hydrophobic groups of the molecule and removing them from the solvent. With docking simulations, we have generated four potential complexes (C1-C4) of AT-amino- $\beta-C D$, in which different groups of AT bind inside the cavity of amino- $\beta-C D$ (Figure 3). It is clear from the proportions of the cavity of amino- $\beta-C D$, that it is impossible to completely bind AT into the amino- $\beta-C D$ cavity. This cavity 
with a diameter and height around $0.6 \mathrm{~nm}$ is capable of binding one of the side-groups of AT emanating from the central pyrrole ring.

In order to quantitatively assess the binding propensity, we calculated the binding enthalpy using the MM-PBSA [Kollman et al. 2000 ; Lynn et al. 2014] protocol. We find that all complexes are thermodynamically stable (see Table 1), but complex C2 has by far the lowest binding energy $\left(-101.63 \mathrm{kcal} \mathrm{mol}^{-1}\right)$, because of a favourable solvation contribution. In C2 the hydrophobic phenylcarbamoyl ring is completely hidden in the hydrophobic amino- $\beta-C D$ pocket while the two other hydrophobic groups are partially covered by the outer amino- $\beta-C D$ ring.

Hydroxypropyl- $\beta$-cyclodextrin is commonly used to deliver drugs. In the AT-hydroxypropyl- $\beta$ CD complex studied by Lv et al (ref Lv-Waddad), molecular modelling studies have shown that the spontaneous inclusion process is realised by the heptanoic acid chain through interaction with the CD primary side hydroxyl groups, and stabilised by the formation of hydrogen bonds and electrostatic interactions. This configuration leaves the 3 phenyl-based substituents still accessible for further complexation, which could explain why the solubility diagram pattern obtained by the authors suggested two different mechanisms of complexation: at low CD concentration the first part of the plot was linear, attributed to a 1:1 complex, whereas the decreased parabolic plateau tendency observed at high CD concentration suggested a higher stoechiometry relative to CD describing a "supermolecule". Hussein et al (hussein) reported for AT/ $\beta-C D$ a deviation from linearity and suggested both $1: 1$ and 1:2 (AT:CD) stoechiometry; similar deviation from linearity was reported by Dey et al (ref dey), for the AT/ $\beta$-CD complex in water, suggesting formation of higher order inclusion complexes ascribed to the formation of complex aggregates (nanoplexes) involving non-inclusion complexation, or micellar aggregates. This phenomenon has been evidenced by Loftsson et al (ref LoftssonJPharmSci202) in a study involving several drugs and several CDs. 
For the 1:1 complex, these authors found a binding energy of $-101.62 \mathrm{kcal} \mathrm{mol}^{-1}$. This low value indicates a quite spontaneous binding process and implies a high stability of the complex. For AT-amino- $\beta-C D$, the binding energy found for the $C 3$ complex (inclusion of the acid chain) is very similar $\left(-106.33 \mathrm{kcal} \mathrm{mol}^{-1}\right)$, but the other complexes $\mathrm{C} 1, \mathrm{C} 2, \mathrm{C} 4$ are slightly more favored, and for the C2 complex we found an even lower value of the binding energy, $-117.39 \mathrm{kcal} \mathrm{mol}^{-}$

${ }^{1}$, indicating an even more spontaneous binding process, in accordance with the corresponding higher stability constant $\left(368 \mathrm{M}^{-1}\right)$, compared to the AT-hydroxypropyl- $\beta$-CD complex $\left(164 \mathrm{M}^{-1}\right.$ reported by Lv or $312 \mathrm{M}^{-1}$ reported by Shivanand, at $25^{\circ} \mathrm{C}$ ) (Lv-waddad, shivanand).

\subsection{SEM/TEM Observations of the Complexes}

As a first step, we observed atorvastatin alone by SEM (Figure 4A). Atorvastatin forms numerous micellar self-assembled aggregates from a few nanometers (visible on the background) to about $50 \mathrm{~nm}$ wide in general, aggregating themselves subsequently at a bigger scale, following two different tendencies: stacking of discoid aggregates with uni-directional tendency, or more spherical-like sprouting (Figure 4B). This has been confirmed by TEM observations (Figure 5A, Figure 5B). The amino- $\beta-C D$ polymer itself is deposited as spherical polymeric globules about 10-20 nm, which tend to aggregate as a network (Figure 4C, Figure 5C). This size is understandable if one considers that the outside diameter of $\beta-C D$ is about 1.5 $\mathrm{nm}$ and the amino- $\beta-C D$ polymer comprises about $15 C D$ units. The circumference $C=2 \pi R$ of such globule would be approximated as $15 \times 1.5 \mathrm{~nm}(23 \mathrm{~nm})$ to which should be added the linking spacers. So for an estimated circumference $C$ of about $30 \mathrm{~nm}$, the radius of the polymeric CD globule would be $4.77 \mathrm{~nm}$, hence a diameter of $9.55 \mathrm{~nm}$. The observation of drops of AT/amino- $\beta$-CD polymer complex displays again a background of globular aggregates, bigger than before, of average size $100 \mathrm{~nm}$, much more evenly distributed in size, which tend to assemble themselves into bigger structures (more than $500 \mathrm{~nm}$ ), again sprouting in a rodlike shape, or a more spherical assembly (Figure 4D, Figure 5D, E, F). Figure 5F nicely displays 
the bridging interactions between basic complex particles, which appear like pincushions. One can also distinguish substructural details in these complex particles, specially from the outside periphery, corresponding to the size of polymeric CD globules (about $20 \mathrm{~nm}$ ). The TEM pictures confirm the basic homogeneous size distribution of the polymeric AT-CD complexes around $100 \mathrm{~nm}$ previously observed by TEM.

Atorvastatin presents amphiphilic characteristics, which explain micellar aggregation properties. It has a hydrophilic tail (dihydroxyheptanoic acid) associated to a large hydrophobic moiety composed of mainly 3 aromatic substituents (phenyl, fluorophenyl, phenylcarbamoyl) as well as a propanyl-pyrrole. The phenylcarbamoyl substituent is actually the combination of a phenyl group linked to a amide bond. Atorvastatin calcium as a solid is a dimer, with strong electrostatic bonds between the acid chains and the calcium ions, as well as other weaker bonds such as hydrogen bonds. This local structure has been evidenced by Wang et al through solid state NMR studies (ref wang 2012), despite the absence of X-ray structure for this solid. The aromatic substituents can be involved in hydrophobic interactions such as $\pi$-stacking: the phenyl and fluorophenyl would preferably face each other to minimize steric hindrance, and thereby could enable further phenyl intercalation from a neighbouring atorvastatin molecule, whereas the phenyl linked to the amide bond is further away and can itself be involved in other hydrophobic or $\pi-\pi$ interactions, also depending on the change in conformation of the amide bond. The aromatic moieties can undergo edge-face, offset stacked or face-to-face stacked interactions. In aqueous media, the hydrophilic and hydrophobic parts of the molecules will tend to self-assemble in order to constitute a hydrophobic core, with the hydrophilic parts pointing out. The levels of freedom from amide bond and acid chain could explain the sprouting effect observed under SEM/TEM. Such self-assembly properties have been described for example by Zhang et 
al (ref zhang 2016) for a series of amide compounds with various aromatic substituent headgroups. Depending on their features they form various "organogels" (supramolecular gels) in various organic solvents, and their self-assembly capacity depends greatly on the size of the aromatic headgroup. These molecules could selfassemble and stack into different shape-like structures such as belt-, rod-, lamellae- or wrinkle-shape structures, depending on solvent environment. These self-assemblies are based on a range of weak interactions, including $\pi-\pi$ stacking, other hydrophobic interactions from aromatic moieties, and hydrogen bonds; these assemblies are often reversible and depend also on $\mathrm{pH}$ and ionic force. A spectacular example is given by Xie et al (ref xie) who designed, in the same family of compounds, a FMOC tripeptide, N-(9-Fluorenylmethoxycarbonyl)-phenylalanine-tryptophan-lysine (Fmoc-Phe-Trp-Lys$\mathrm{OH})$, which formed nanofibers at $\mathrm{pH}$, flat ribbons at $\mathrm{pH}$ 6-11 and helical nanoribbons above $\mathrm{pH} 11$, the latter assemblies being based on a more rigid conformation of the peptide with strong intramolecular $\pi-\pi$ interaction between the tryptophan and the Fmoc moieties.

The amino- $\beta-C D$ polymer is produced from a cross-linking reaction with epichlorhydrin between 6-monodeoxy-6-monoamino- $\beta$-CD units, which can occur on primary or secondary side. All amino substituents can make hydrogen bonding with hydroxyl protons either from water or from neighbouring $C D$ units, which tends to condensate the network. This could explain why we can see by SEM formation of polymeric spherical nanoparticles of about 10 $\mathrm{nm}$. This size is in accordance with the average $15 \mathrm{CD}$ units on a polymeric molecule. These spherical particles can aggregate into bigger assemblies and tend to form a reticulate network.

The bigger particulate size observed for AT/amino- $\beta$-CD polymer complexes (about $100 \mathrm{~nm}$ basic units) is also in accordance with the Tyndall effect (scattering of light by particles in suspension) we observed for the aqueous solution of amino- $\beta-C D$ polymer due to particulate 
suspension, effect which was increased upon complexation, suggesting the formation of larger aggregates.

Comparable supramolecular assemblies have been reported by Yang et al based on the synthesized polymer per-methyl- $\beta-C D$ modified hyaluronic acid $(\mathrm{HA})$, complexing the pro-drug adamplatin (adamantyl groups) (ref yang 2013), or more recently, complexing the porphyrin modified paclitaxel pro-drug through strong host-guest interaction between the porphyrin and the CD units (ref yang 2016). This led to supramolecular amphiphilic complexes self-assembling into structurated nanoparticles with a hydrophilic HA outer shell (able to target HA-receptor on cells) and a hydrophobic pro-drug core. These nanoparticles were biocompatible, biodegradable and lowered the side-effects of the paclitaxel anti-cancer drug. This HA-pCD polymer displays a molecular weight (28000) similar to our amino- $\beta-C D$ polymer and an average hydrodynamic radius around $20 \mathrm{~nm}$, also comparable. The complexes formed spherical nanoparticles of $30-50 \mathrm{~nm}$, tending to self-aggregate in bigger assemblies through hydrogen bonds interactions between particles (carboxyls ans hydroxyls of the HA), resulting in a measured hydrodynamic radius of $171 \mathrm{~nm}$, with a zeta potential of $-29 \mathrm{mV}$ (negative, from $\mathrm{HA}$ acids). This is all quite comparable to our results, as AT/amino- $\beta-C D$ polymer complex forms globular aggregates of average size $100 \mathrm{~nm}$, which tend to aggregate into bigger structures (more than $500 \mathrm{~nm}$ ), probably due to inter-particle amino/hydroxyl hydrogen bonds from the CD units; moreover we measured a zeta potential of +14 to $+27 \mathrm{mV}$ of the particulate complexes, which confirms the amino nature $(+)$ of the outer shell.

\subsection{SEM Observation of Gel Properties}

Finally, we would like to point out that in certain conditions of SEM/TEM preparations, with highly concentrated amino- $\beta-C D$ polymer (with or without AT), the polymer network changed into a hydrogel upon rinsing the glass- or copper-adsorbed sample with water. This hydrogel structure was photographed by SEM and displayed on Figure 6 . Under the electron beam, it 
quickly made unidirectional wrinckles followed by apparition of pores $(250-750 \mathrm{~nm})$, as the water was replaced by gas. It is quite comparable to pictures obtained by Aouada et al with poly-acrylamide (Aouada 2005) or El Fray (El Fray 2007) with a cryostructured poly(vinyl alcohol) hydrogel: the tuned cross-linking and process cycles related hydrogel morphology resulted in highly porous (pore size 1-2 $\mathrm{mm}$ ) and ribbon-like, oriented structure, presenting adequate mechanical properties compared to cartilage. Aikawa et al (Aikawa 1998) described a behaviour similar to ours for polyvinylacetal diethylaminoacetate from solution at $\mathrm{pH} 4$ to porous hydrogel at pH 7.4 and its drug release properties; so did Pourjavadi et al (Pourjavadi 2007) for a superabsorbent hydrogel resulting from copolymerisation of acrylamide/sodium acrylate and hydrolised collagen, showing high sensitivity to $\mathrm{pH}$ inducing reversible swelling, and rendering this hydrogel a good candidate for drug delivery.

The results shown here suggest another potential application of epichlorhydrin crosslinked amino- $\beta-C D$ polymer (loaded or not with drug), as cartilage mimics. As a new potential physical hydrogel stabilised by weak bonds such as Van der Waals, hydrogen or ionic bonds, it can be used as replacement material for cartilage, with advantages such as appropriate physical properties, good transport of nutrients and cell products via pores, biocompatibility, reversible sol-gel properties.

\subsection{SEM Observation of the Functionalised Membranes}

Figure 7 displays functionalised PCL-PLL-DEX-(CPLX-DEX- $)_{n}$ membranes $(n=5$ or 10$)$, together with the bare control membrane (Figure 7A), and PCL-PLL-DEX-(CD-DEX-) for comparison. A lot of nanoreservoirs are visible, evenly spread over the surface of the membrane's fibres, as can be seen with low concentration of CD-polymer (190 $\left.\mathrm{g} \mathrm{mL}^{-1}, \mathrm{n}=5\right)$ (Figure 7B). When increasing the CD-polymer concentration (to $1 \mathrm{mg} \mathrm{mL}^{-1}, \mathrm{n}=5$ ), the reference coating (Figure $7 \mathrm{C}$ ) covers the whole surface of the fibers and displays the classical nanoreservoirs, whereas in the

presence of AT complexes (in same conditions) (Figure 7D) we see the beginning of 
supplementary lateral interactions, leading to the onset of coating extensions. This effect of lace-like extensions in particular at crossings is further enhanced when increasing the number of cycles ( $1 \mathrm{mg} \mathrm{mL}^{-1} \mathrm{CD}$-polymer, $\mathrm{n}=10$ ) (Figure $7 \mathrm{~F}$ compared to $\mathrm{E}$ ). This propensity to lateral extensions ultimately leads to the formation of interfiber films. Thus, the studied system not only allows the formation of homogeneously dispersed active nanoreservoirs, but also the formation of thin active films, which could bring other particular applications.

In the nanoreservoirs, composed of the different compounds sequentially adsorbed during the functionalisation process, the molecule of interest, atorvastatin, should be present as complexes, but to prove that point, and to try to quantify the atorvastatin present in the membrane, we performed XPS measurements on the membranes and spectrophotometric quantification of atorvastatin embedded in the membranes.

\subsection{XPS Measurements on the Functionalised Membranes}

The chemical composition of the membranes surface was obtained by XPS. Spectra of all membranes reveal the presence of atoms $\mathrm{C}, \mathrm{O}, \mathrm{N}, \mathrm{S}, \mathrm{Na}$ and $\mathrm{F}$ on the surface. The relative atomic concentrations were calculated from the area of the main signal of the observed elements with appropriate atomic sensitive factors and after Shirley background subtraction.

Polyatomic ion beam sputtering with XPS analysis provided quantitative chemical state information as a function of depth with minimal ion beam induced chemical damage. A $10 \mathrm{kV}$ $\mathrm{Ar}^{+}$cluster source was rastered over $2 \mathrm{~mm} \times 2 \mathrm{~mm}$ area to create the depth profile (with an approximate sputter rate of $95 \mathrm{~nm} / \mathrm{min})$. A $110 \mu \mathrm{m} \times 110 \mu \mathrm{m}$ spot located in the center of the rastered square was analyze.

The variation of fluor (F) atomic concentration with etching time was measured in five different PCL-PLL-DEX-(CPLX-DEX-) $)_{5}$ membranes and the results are shown in Figure 8 . This element is related to the presence of atorvastatine in the samples, and according with the 
results it would be slightly more concentrated on the surface but its signal remains unchanged in the membrane bulk. This is in accordance with SEM pictures which show the presence of the nanoreservoir coating deep inside the nanofibrous membrane.

\subsection{Spectrophotometric Quantification of Atorvastatin Embedded in the Membranes}

\subsubsection{Dissolution with methanol}

To quantify the atorvastatin present in the membrane we tried to dissolve the embedded molecule with successive washings of the membrane into methanol (Figure 9A). The UV-vis spectrum of the first recovered methanol solution, diluted with equivalent volume of MES buffer, displayed the features of atorvastatin. Then, in subsequent methanol washings spectra, there is less and less atorvastatin recovered, until a few traces only. The quantity of atorvastatin was determined for each washing (Overnight, vortex 1, 2, 3 and 4) using the calibration curve and then summed up to have the total quantity of atorvastatin dissolved out of the membrane. The resulting average (of 3 experiments) was $26( \pm 22) \mu g$ for 5 cycle membranes, and 30( \pm 21$) \mu \mathrm{g}$ for 10 cycles membranes.

\subsubsection{Analysis of the dipping solutions of complex}

For a concentration of amino- $\beta-C D$ polymer of $1 \mathrm{mg} \mathrm{mL}^{-1}$, the concentration of atorvastatin solubilised in MES is $200 \mu \mathrm{g} \mathrm{mL} L^{-1}$ according to the previous results. The volume of dipping complex solution poured in the well (96 well plate) was $300 \mu \mathrm{l}$ so the quantity of atorvastatin in solution in the well containing the membrane is initially $60 \mu \mathrm{g}$.

From the UV spectra of the complex dipping solutions used for membrane functionalisation, we determined the quantity of atorvastatin remaining in the dipping solutions after adsorption and, by subtraction with the initial quantity present in the dipping solution, calculate the quantity adsorbed in the membrane. 
The total quantity of atorvastatin adsorbed on the membrane was obtained by the addition of the quantities adsorbed at each cycle. As an average of 4 experiments, the quantity of atorvastatin adsorbed on the membrane was $84 \pm 11 \mu \mathrm{g}$ for 5 cycles membranes, and $200 \pm 13$ $\mu \mathrm{g}$ for 10 cycles membranes.

\subsubsection{Comparison of the two approaches}

We have been able to estimate the quantity of atorvastatin present on the membrane by two different approaches.

The limits of the first method are the uncertainty concerning the dissolution of all the atorvastatin present on the membrane, as well as the possible variation in membrane thicknesses. The first point is suggested by the levelling of quantity dissolved with methanol despite the increase from 5 to 10 cycles of adsorption. Concerning the membrane itself, its thickness may differ slightly from one to another, yet the quantity absorbed on one membrane may be higher if the membrane is thicker than another, or the contrary if the membrane is thinner.

The dispersion of the results with the second approach is much smaller than with the methanol dissolution method. Another big difference is the expected increase in membraneembedded atorvastatin (complex) when going from 5 cycles to 10 cycles of adsorption. The fact that the quantity is more than doubled is actually in accordance with the increasing occurence of extended lace networks in the coating, observed by SEM, when going from 5 to 10 cycles of adsorption. Finally, we know how much atorvastatin we have at the end of the functionalisation but we need to know if the molecule of interest remains on the membrane when left in the buffer after the functionalisation.

\subsubsection{Follow-up of potential atorvastatin release}


The recording of UV-vis spectra of MES buffer at each rinsing cycle during functionalisation showed that a tiny amount of adsorbed atorvastatin (about $1 \mu \mathrm{g}$ ) is washed out of the membrane at each first of the two washings. As demonstrated by the absence of atorvastatin characteristics in the UV-Vis spectra of the MES buffer above the membrane, taken after the functionalisation, no release of Atorvastatin out of the membrane is observed even 2 days after. The functionalised membranes can this way be stored probably a few months without noticeable change, as was confirmed by XPS measurements.

The $1 \mu \mathrm{g} / \mathrm{cycle}$ loss during functionalisation has to be subtracted from the quantity of embedded drug determined from dipping complex solutions, which leads to doses of $79 \pm 11 \mu \mathrm{g}$ for 5 cycles membranes, and $190 \pm 13 \mu \mathrm{g}$ for 10 cycles membranes.

When soaking the functionalised membrane from MES into PBS pH 7.4 buffer, the UV-vis spectra of that rinsing buffer taken at various times in the first 48 hours show that there is a little bit of release of atorvastatin in the first hour (Figure 9B, see above $250 \mathrm{~nm}$ ) although in small amount, estimated to 3-4 $\mu$ g i.e. about $6 \%$ loss, which has to be subtracted also when expressing final dose at $\mathrm{pH} 7.4$ for in vitro/in vivo studies.

\subsection{PCL Membranes Embedded with Atorvastatin Repress TNF- $\alpha$ and IL-6 Production in LPS- activated THP-1 Cells.}

We evaluated the anti-inflammatory properties of atovastatin, which, for potential future use in rheumatic diseases, has been embedded into a biodegradable matrix of polycaprolactone $(\mathrm{PCL})$ that might be inserted into the synovial space of affected joints. The embedding into the nanoreservoirs should enable delayed and long-term consumption of the active molecule, as it is a cell contact-dependent delivery mode through degradation of the excipent polymer by the contacting cells (Mendoza-acsNano2012). The aim of the present study was to investigate the impact of the PCL membranes loaded with the anti-inflammatory molecule on cytokine expression. For this, empty and statin-embebbed PCL were seeded in culture wells with 
control or LPS-stimulated THP-1 cells and TNF- $\alpha$ and IL-6 expression were monitored over time. The model is studied from 6 to $48 \mathrm{~h}$. We first noticed that empty PCL membranes do not induce cytokine expression in control cells, nor do they repress IL-6 or TNF- $\alpha$ secretion by LPSactivated cells. Next, as seen in Figure 10, we observed that both TNF- $\alpha$ (panel A) and IL-6 (panel B) secretion in the medium is reduced when cells are cultured on atorvastatincontaining PCL membranes, at late time points ( 24 and $48 \mathrm{~h}$ post LPS stimulation), while the atovastating-containing PCL membrane appear inefficient on early (6h) time points, when TNF$\alpha$ secretion is at its maximal levels. This effect is also visible on IL-6 expression, which is characterized by a slower secretion with peak values at $48 \mathrm{~h}$. Rather than suggesting a limited efficacy of atovastatin-coated PCL membranes, this observation indicates that the statin delivery from the biomaterial is delayed. Alternatively, it is also conceivable that atorvastatindependent post-traductional modifications of signaling proteins such as Ras/GTPases acting downstream of TLR4 might initially exhibit only minor effects on cytokine expression, but whose consequences would result in the amplification of an inhibitory signal.

Concerning TNF- $\alpha$ secretion, increasing the "dose" when using complexes from $10 \mathrm{mg} \mathrm{mL}^{-1}$ cyclodextrin polymer (Condition 1), instead of $1 \mathrm{mg} \mathrm{mL}^{-1}$ (Condition 2), has no supplemental effect on inflammation inhibition, at any time point, reaching about $40 \%$ inhibition at $48 \mathrm{~h}$. Interestingly, the effects of PCL-embedded atorvastatine appear dose-dependant with regards to IL-6 secretion, with a higher effect for the Condition 2 functionalisation, with a maximum of 74.9\% inhibition of IL-6 at $48 \mathrm{~h}$. Preliminary experiments with varying the number of drug complex adsorption cycles $\left(\mathrm{NR}_{\mathrm{n}}\right)$ for the membrane functionlisation, with $\mathrm{n}=2$ or 5 , have shown that there were no noticeable difference, so $n=2$ was estimated as sufficient and chosen for all experiments.

Future experiments will be necessary to decipher in more details the pathways that are disrupted by atorvastatin. Furthermore, in vivo experiments will be performed to evaluate the 
impact of implanted atorvastatin-embedded PCL membranes. The mouse air pouch model appears as the most advantageous system for this purpose (Dalmarco et al., 2012).

\section{Conclusions}

In this study, we have developed a therapeutic wound dressing with anti-inflammatory properties, consisting in a synthetic nanofibrous electrospun polycaprolactone membrane functionalised with nanoreservoirs containing atorvastatin in the form of inclusion complexes with a polymeric cyclodextrin. We first examined the behaviour of this complex system in solution and demonstrated the complex formation through fluorescence and UV-vis spectroscopy, corroborated by MD simulations. Further characterisation on the supramolecular organisation of this complex system was obtained from SEM/TEM observations and is discussed in terms of the chemical structure and properties of the compounds. Polyamino- $\beta$-cyclodextrin was chosen to achieve a double objective: (i) to solubilise the poorly soluble hydrophobic atorvastatin through formation of inclusion complexes in the cyclodextrin cavities and/or nanoplexes, (ii) to be suitable for the build-up of the nanoreservoirs, as it is a positively charged polyelectrolyte. Nanoreservoirs were formed through alternate adsorption of polyamino- $\beta$-cyclodextrin-atorvastatin and dextran sulfate. The functionalised membranes were analysed by SEM to confirm formation of the nanoreservoirs on the membrane fibers. Different approaches were used to confirm the presence of the drug in the nanoreservoirs, and estimate its quantity and release behaviour. XPS measurements on the membranes with etching showed that atorvastatin (specific $F$ atom) is present uniformously in the membrane bulk. UV-vis measurements after methanol washings (extraction of the drug from the membrane) and analysis of solutions used for the nanoreservoirs build-up (remaining drug, non-adsorbed), lead to an estimation of the drug 
present on the membranes. Pros and contras of the two approaches are discussed. UV-vis follow-up of the buffer after the build-up showed no release in MES, and a slight release when going into PBS (6\% loss), and this stability of the functionalised membranes was confirmed by XPS measurements after a few months, still showing the atorvastatin signal. This behaviour enables to get a small release of the drug at the time of the membrane insertion at the lesion site (physiological $\mathrm{pH}$ ), and after that the delivery of the drug is cell contact dependent, as in other applications of the patented nanoreservoirs. Upon contact, cellular enzymes degrade the polymer coating and the drug becomes available; as cells grow they infiltrate deeper in the porous scaffold inducing the sustained release of the drug. In a model system, the designed atorvastatin-functionalised wound patches were seeded with control or LPS-stimulated THP-1 macrophages and we monitored inflammatory cytokine expression (TNF- $\alpha$ and IL-6).

TNF- $\alpha$ inhibition reaches about $40 \%$ at 48 h (no dose effect), and up to $74 \%$ for IL-6, depending on the dose. Future experiments will be done to investigate in more details the pathways disrupted by atorvastatin. Furthermore, in vivo experiments will be performed to evaluate the impact of the functionalised wound patches, such as the mouse air pouch model, for rheumatic application, and the mouse periodontal wound pocket, for periodontal application. The novelty of the system resides in the double protection offered by the cyclodextrins as drug molecular chaperones, combined with further embedding into cell contact-dependent biodegradable nanoreservoirs. As the designed wound dressing is a highly versatile therapeutic platform, it is likely to be applied for instance as a dual atorvastatin/BMP-2 delivery system for bone regeneration and inflammation modulation in periodontal repair.

\section{Experimental Section}




\section{Experimental Subheading: Experimental Details. 12 point, double-spaced.}

\section{References are superscripted and appear after the punctuation. ${ }^{[6]}$}

Physical data should be quoted with decimal points and negative exponents (e.g.,

$25.8 \mathrm{~J} \mathrm{~K}^{-1} \mathrm{~mol}^{-1}$ ), and arranged as follows where possible: $\mathrm{mp} / \mathrm{bp} 20^{\circ} \mathrm{C} ;[\alpha]_{\mathrm{D}}{ }^{20}=-13.5$ ( $c=0.2$, acetone) (please also give units for $[\alpha]$ and $c$, usually $\mathrm{deg}_{\mathrm{cm}} \mathrm{g}^{-1} \mathrm{dm}^{-1}$ and $\mathrm{g}$ $\mathrm{cm}^{-3}$, respectively); ${ }^{1} \mathrm{H}$ NMR (400 MHz, DMSO- $d_{6}, \delta$ ): 7.15 (s, 2H, Ar H), 1.3 (q, $J=$ $\left.8 \mathrm{~Hz}, 2 \mathrm{H} ; \mathrm{CH}_{2}\right), 0.9$ (t, $\left.J=8 \mathrm{~Hz}, 3 \mathrm{H} ; \mathrm{CH}_{3}\right) ;{ }^{13} \mathrm{C} \mathrm{NMR}\left(100 \mathrm{MHz}, \mathrm{CDCl}_{3}, \delta\right): 175.4$ $(\mathrm{C}=\mathrm{O}), 156.5(\mathrm{C} 4)$; IR (KBr): $v=2972(\mathrm{w}), 2907(\mathrm{w}), \ldots, 1026\left(\mathrm{~s} ; v_{\mathrm{as}}(\mathrm{SiOSi})\right), 971$ $\left(v_{\mathrm{s}}\right), \ldots, 666\left(\mathrm{w} ; v_{\mathrm{s}}(\mathrm{SiOSi})\right), \ldots, 439(\mathrm{~m}), 401 \mathrm{~cm}^{-1}(\mathrm{~m})$; UV-vis $\left(n\right.$-hexane) $: \lambda_{\max }(\varepsilon)=$ 320 (5000), 270 nm (12000); EIMS m/z (\%): $108(20)\left[\mathrm{M}^{+}\right], 107(60)\left[\mathrm{M}^{+}-\mathrm{H}\right], 91$ (100) $\left[\mathrm{C}_{7} \mathrm{H}_{7}{ }^{+}\right]$; HRMS (ESI) $m / z$ : $[\mathrm{M}+\mathrm{H}]^{+}$calcd for $\mathrm{C}_{21} \mathrm{H}_{38} \mathrm{~N}_{4} \mathrm{O}_{6} \mathrm{~S}, 475.2591$; found, 475.2593. Anal. calcd for $\mathrm{C}_{45} \mathrm{H}_{28} \mathrm{~N}_{4} \mathrm{O}_{7}$ : C 62.47, $\mathrm{H} 3.41, \mathrm{~N} 6.78$; found: $\mathrm{C} 62.27, \mathrm{H}$ $3.46, \mathrm{~N} 6.80$.

[CCDC \#\#\#\#\# contains the supplementary crystallographic data for this paper. These data can be obtained free of charge from The Cambridge Crystallographic Data Centre via www.ccdc.cam.ac.uk/data_request/cif.] ((delete if not applicable))

[Further details of the crystal structure investigation(s) may be obtained from the Fachinformationszentrum Karlsruhe, 76344 Eggenstein-Leopoldshafen (Germany), on quoting the depository number CSD-...] ((delete if not applicable))

Reagents: Atorvastatin calcium trihydrate was purchased from Sigma-Aldrich, France. Amino$\beta$-cyclodextrin polymer (abbrev. amino- $\beta-C D$ polymer), namely 6-Monodeoxy-6-monoamino$\beta$-cyclodextrin soluble polymer crosslinked with epichlorohydrin (Av. MW $25 \mathrm{kDa}$ ) was 
purchased from Cyclolab, Hungary. 2-(N-morpholino) ethanesulfonic acid (MES) from SigmaAldrich (France), and sodium chloride from Euromedex (France), were used to prepare MES buffer at pH 5.5 (40 mM MES, $150 \mathrm{mM} \mathrm{NaCl);} \mathrm{Methanol} \mathrm{(Abbrev.} \mathrm{MeOH)} \mathrm{from} \mathrm{Prolabo} \mathrm{(France)}$ was used as miscible organic solvent in solubilisation experiments. Poly( $\varepsilon$-caprolactone) (Abbrev. PCL) clinical grade, MW 124 kDa, was obtained from Purac (Gorinchem, Netherlands). Other reagents used for electrospinning were dichloromethane and dimethylformamide from Sigma-Aldrich (France). Polyelectrolytes used for the functionalisation of PCL electrospun membranes were poly(L-lysine) (Abbrev. PLL) and dextran sulfate (Abbrev. DEX) from SigmaAldrich, France.

Cell culture media (RPMI 1640), FCS, L-glutamine, penicillin, streptomycin and amphotericin B were from Invitrogen (Cergy-Pontoise, France). LPS from Salmonella abortus equi was obtained from Sigma (Saint-Quentin-Fallavier, France). The enzyme immunoassay kits for human TNF- $\alpha$ and IL-6 detection were from Bio-techne (Lille, France).

\section{Spectrophotometric complexation and solubilisation studies.}

Solubilisation of Atorvastatin by amino-6-cyclodextrin polymer: A range of increasing concentrations of amino- $\beta-C D$ polymer, from 0.08 to $10 \mathrm{mg} \mathrm{mL}^{-1}$, was prepared in MES buffer in $2 \mathrm{~mL}$ Eppendorf tubes. In each tube was added $1 \mathrm{mg}$ of atorvastatin excess powder and the mixtures were shaken overnight. Atorvastatin is sparingly soluble in aqueous medium, and reported solubility values depend on authors and conditions, ranging from $4.8 \mathrm{mg} \mathrm{mL}^{-1}$ (Dey2014) or $27.04 \mathrm{mg} \mathrm{mL}^{-1}$ (Narasaiah 2010) in distilled water $25^{\circ} \mathrm{C}$, to $57.06 \mathrm{mg} \mathrm{mL}^{-1}$ in PBS pH 6.8 (Narasaiah 2010), or to even higher values such as $120 \mathrm{mg} \mathrm{mL}^{-1}$ in water (Hussein 2011) or $149.3 \mathrm{mg} \mathrm{mL}^{-1}$ as saturation solubility (Kulthe 2013). Soluble amino- $\beta-C D$ polymer will increase its solubility by forming inclusion complexes. The concentration of drug solubilised is 
determined by UV-vis spectroscopy, from a calibration curve established for atorvastatin in $\mathrm{MeOH}$ :MES (1:1). As the drug is sparingly soluble in aqueous medium, but soluble in organic solvent like methanol, we chose this mixed solvent for the calibration curve. For the measurement of absorbance, the excess atorvastatin powder in the mixtures was filtered off, and each mixture supernatant was mixed at equal volume with methanol in order to be in the same conditions as used for the calibration curve of atorvastatin in MeOH:MES (1:1). From the concentration of atorvastatin determined in $\mathrm{MeOH}: \mathrm{MES}$, the concentration of drug initially dissolved by amino- $\beta-C D$ polymer in MES was obtained as the two-fold. The measure of absorbance was done between 200 and $350 \mathrm{~nm}$, on UV-Vis Spectrophotometer Uvikon XL (BioTek Instrument, USA) with Lab Power Junior software. $1 \mathrm{~cm}$ path quartz cuvettes (Hellma) were used. A calibration curve was performed in MeOH:MES (1:1) prior to these experiments, with atorvastatin concentrations ranging from 0.01 to $2.5 \mathrm{mg} \mathrm{mL}^{-1}$.

Fluorescence spectroscopy: Fluorescence properties of atorvastatin in the absence and presence of increasing quantities of amino- $\beta-C D$ polymer (in MES pH 5.5) were examined as a means of evaluation of the drug microenvironment. Indeed, an increase in the fluorescence intensity of similar hydrophobic drugs upon addition of $C D$ was often used as a demonstration of the inclusion of the drug in the CD cavity, as the hydrophobic micro-environment provided by this inclusion boosts the fluorescence yield of the drug. Spectra were measured on a Spectracq Fluorolog fluorescence spectrometer. Excitation was set at $276 \mathrm{~nm}$, and emission was measured between 300 and $500 \mathrm{~nm}$ (emission peak of atorvastatin is at about $381 \mathrm{~nm}$ ), with a $5 \mathrm{~nm}$ slit and integration time $1 \mathrm{~s}$. Mixtures of atorvastatin/amino- $\beta-C D$ polymer in MES were prepared in $2 \mathrm{ml}$ Eppendorf tubes, at constant concentration of drug $\left(1 \mu \mathrm{gL}^{-1}\right)$ and increasing concentration of polymer (from $93 \mu \mathrm{g} \mathrm{mL}^{-1}$ to $3.75 \mathrm{mg} \mathrm{mL}^{-1}$ ) and flurescence spectra measured between 300 and $500 \mathrm{~nm}$.

Quantification of Atorvastatin contained in functionalised membranes : Two approaches were used to try and quantify atorvastatin in the membranes. In a first approach, the 
functionalised membrane was soaked into methanol overnight, in order to solubilise the adsorbed atorvastatin. The next day, the solution of methanol containing the dissolved atorvastatin was mixed with an equal volume of MES buffer and the absorbance was read with a UV spectrophotometer, between $200 \mathrm{~nm}$ and $350 \mathrm{~nm}$. Afterwards, in the Eppendorf tube containing the membrane, fresh methanol was added and the tube was vortexed to be sure that a maximum of the drug had been removed from the membrane, then the methanol was taken and again mixed with an equal volume of MES buffer and the absorbance read (Vortex 1). This process was repeated a few times (Vortex $2,3,4$ ), as long as an atorvastatin spectrum was recognisable. Quantification of the drug recovered from the membrane was performed by use of the calibration curve and by summing up the amounts obtained from the successive methanol washing steps. In a second approach, the drug-CD complex solution, used at each adsorption step during the functionalisation of the membrane, was kept and put in an Eppendorf tube, and mixed with an equal volume of methanol. The measure of the absorbance was done between 200 and $350 \mathrm{~nm}$. The idea was to estimate how much drug had been adsorbed at each step by measuring what was left in the dipping solution. Again, the quantification was performed by use of the calibration curve and by summing up the amounts obtained from each adsorption step.

Quantification of Atorvastatin release from PCL membranes: After functionalisation of the membrane, we have checked the potential release of atorvastatin from the membrane, comparing two different buffers: MES ( $\mathrm{pH}$ 5.5) used during functionalisation, and PBS buffer at $\mathrm{pH} 7.4$, for the in vitro conditions of use of the membrane in the presence of cells and cell culture medium. After functionalisation, membranes were left to rest in a well, either in MES or in PBS buffer. Buffer was removed and replaced with fresh one, at various times ( $2 \mathrm{~h}, 18 \mathrm{~h}$, $20 \mathrm{~h}, 24 \mathrm{~h}$ and $48 \mathrm{~h}$ after the end of the functionalisation). The recovered buffer solutions were then mixed with an equal volume of methanol, and the absorbance a UV spectrophotometer, 
between $200 \mathrm{~nm}$ and $350 \mathrm{~nm}$. Quantification of potentially released atorvastatin was performed by the means of calibration curves, in MES:MeOH and in PBS:MeOH (1:1).

\section{Preparation, characterisation and functionalisation of PCL membranes.}

Electrospinning: The fabrication of the membranes was realised with the standard Electrospinning Apparatus EC-DIG provided by IME Technologies (Amsterdam, Netherland). This apparatus is composed of a hermetic enclosure connected to an aeration for chemical extraction, a syringe-pump and its command, a vertical support in order to maintain a $21 \mathrm{G}$ needle $(0.8 \mathrm{~mm}$ of diameter) at a certain distance from a conductive collector of $10 \mathrm{~cm}$ of diameter. The range of voltage furnished by the generator embedded in the apparatus is +25 $\mathrm{kV} /-25 \mathrm{kV}$ (needle/collector). The generator is controlled with a touch screen. This apparatus allow us to elaborate nanofibrous and microporous thin and thick membranes. The manufacture of electrospun thin membranes was done from a solution of poly $(\varepsilon-$ caprolactone) polymer (PCL 124000 ) at a concentration of $27 \%$ (27 mg PCL for $100 \mathrm{~mL}$ of solution) in a mixed solvent composed of dichloromethane (DCM) and dimethylformamide (DMF), at a ratio $40 / 60(\mathrm{v} / \mathrm{v})$. This solution is injected through the needle from a $5 \mathrm{~mL}$ syringe placed in the syringe-pump of the apparatus, via a polydimethylsiloxane tube (PDMS). The solution of PCL is electrospun during 15 minutes, under a $+22 /-3 \mathrm{kV}$ differential voltage, $1 \mathrm{~mL} / \mathrm{h}$ flow rate, $24^{\circ} \mathrm{C}$ and $45 \pm 5 \%$ relative humidity. The fibres are collected on the round collector placed at more or less $16 \mathrm{~cm}$ from the injection needle. At the end of the process, the membrane is put in a heat chamber at $50^{\circ} \mathrm{C}$ to eliminate the remaining solvent from the electrospinning process.

Functionalisation of the electrospun PCL membranes: The technique used for the functionalisation of the membrane is the nanoreservoir technology developed in the laboratory (MendozaACS nano) initially based on the layer-by-layer technique described by G. Decher (Decher 1997) to form multilayer coatings from electrostatic interactions between 
polyelectrolytes of opposite charges, through alternate dippings. The electrospun membrane formed on aluminium foil was cut into small pieces using a round punch $(6 \mathrm{~mm}$ diameter, JLB340P BOEHM ${ }^{\circledR}$, France). To separate the membranes from their aluminium counterpart, they were immersed in a 70\% alcohol bath, and left there for at least one hour. This enabled at the same time some hydrophilisation of the PCL membranes. Afterwards, these were placed at the bottom of wells in a 96 well-plate.

For this membrane functionalisation, pH 5.5 MES buffer (40 mM, with $\mathrm{NaCl} 150 \mathrm{mM}$ ) was used to make the polyelectrolytes solutions and wash out the non-adsorbed polyelectrolytes after each membrane dipping step. We used dextran sulfate $\left(500 \mu \mathrm{g} \mathrm{mL}^{-1}\right)$ as polyanion. The polycation was amino- $\beta-C D$ polymer/atorvastatin complex solution. To prepare this solution, we first dissolved amino- $\beta-C D$ polymer in MES buffer and stirred overnight. We then added an excess of atorvastatin powder to the $\mathrm{CD}$ solution $(0.5 \mathrm{mg}$ for $2 \mathrm{ml}$ of solution). The mixture was vortexed and then shaken for $24 \mathrm{~h}$; after that the excess solid remaining was filtered off and the supernatant solution containing amino- $\beta-C D$ polymer/atorvastatin complex used as polycation dipping solution for the nanoreservoirs formation.

The wells containing PCL membranes were filled with $300 \mu \mathrm{L}$ of MES buffer and left to equilibrate overnight at $4^{\circ} \mathrm{C}$ before beginning the experiment. The functionalisation of the membranes was then realised through successive baths $(300 \mu \mathrm{L})$ of alternate polycation/polyanion dipping solutions. After each polyelectrolyte adsorption step, the dipping solution was removed with a pipette and replaced by buffer, as a rinsing step to remove all non-adsorbed compound ( 2 washings). Each bath lasted $15 \mathrm{~min}$, except for the complex solution (half an hour, necessary to reach a plateau, as determined by preliminary QCM experiments). The build-up was initiated with a standard PLL $\left(500 \mu \mathrm{g} \mathrm{mL}^{-1}\right) / D E X\left(500 \mu \mathrm{gL}^{-1}\right)$ adsorption cycle, as a "primer layer". The construction can be described as -PLL-DEX-(CPLXDEX- $)_{n}$ where CPLX is the solution of amino- $\beta$-CD polymer/atorvastatin complex, and $n$ is the 
number of adsorption cycles, which governs the size of the nanoreservoirs. When the functionalisation is finished, the membranes are left into MES buffer, the plates are stored in the fridge at $4^{\circ} \mathrm{C}$.

SEM/TEM observations: Samples of compounds and complexes in solution were prepared for SEM observation by depositing a drop of solution on a thin microscope glass slide cover, let it dry, repeat the operation a few times, and finally wash out the buffer salt by rinsing the slide with water. These glass covers were then fixed on SEM sample holders and coated for SEM observation.

Functionalised membranes need to be treated and dehydrated before the observation at the SEM. After the functionalisation, the membranes were rinsed three times (10 min each) with phosphate buffer (PB), fixed with $2.5 \%$ glutaraldehyde diluted into PB (10 $\mathrm{min}$ ) at room temperature, and washed three times (10 $\mathrm{min}$ ) with PB. Dehydration was done by successive dippings (10 min each) into ethanol at increasing concentration: $25 \%, 50 \%, 75 \%, 90 \%$, and 100\%. After a quick dipping into HDMS, the membranes were fixed on a sample holder with double coated conductive adhesive tape, and metal-coated ( $\mathrm{Au} / \mathrm{Pd})$ or carbon-coated. SEM observations were made on a Hitachi 5800 scanning electron microscope (Hitachi, Tokyo, Japan), at $5 \mathrm{kV}$ for $\mathrm{Au} / \mathrm{Pd}$-coated samples, and $15 \mathrm{kV}$ for carbon-coated samples. For higher magnification some samples were further observed on a Hitachi TM 1000 (IGBMC).

TEM observations were made with a Hitachi H7500 transmission electron microscope (Hitachi, Tokyo, Japan) equipped with an AMT Hamamatsu digital camera (Hamamatsu Photonics, Hamamatsu City, Japan). Solution samples of atorvastatin, polymer-CD and complexes were prepared for TEM by depositing a microdrop of solution on a TEM grid covered with a carbon film, let it dry, repeat the operation a few times, and finally wash out the buffer salt by rinsing the slide with water. 
XPS analysis: The X-ray photoelectron analysis (XPS) was performed with an Axis Supra (Kratos Tech.). The spectra were excited by the monochromatized AlK $\alpha$ source (1486.6 e.V) run at 15 $\mathrm{kV}$ and $15 \mathrm{~mA}$. For the individual peak regions pass energy of $40 \mathrm{eV}$ was used. Analyses of the peaks were performed with the CasaXPS software after background subtraction 

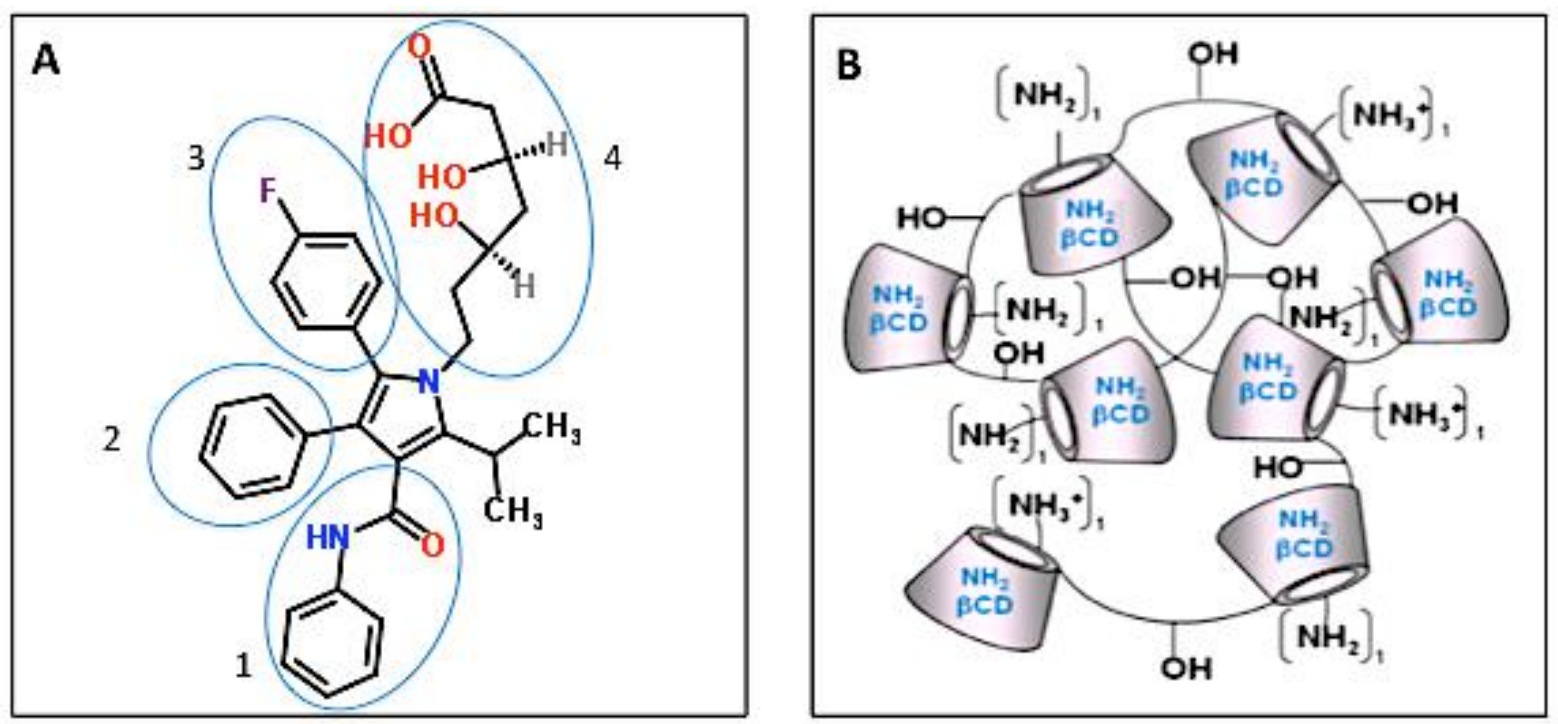

Figure 1. Schematic representation of (A) Atorvastatin and its main chemical groups, 1: phenylcarbamoyl-, 2: phenyl-, 3: fluorophenyl, 4: 3,5-dihydroxy heptanooic acid; (B) The amino- $\beta$-CD polymer produced from a cross-linking reaction with epichlorhydrin between 6monodeoxy-6-monoamino- $\beta$-CD units, which can occur on primary or secondary side.
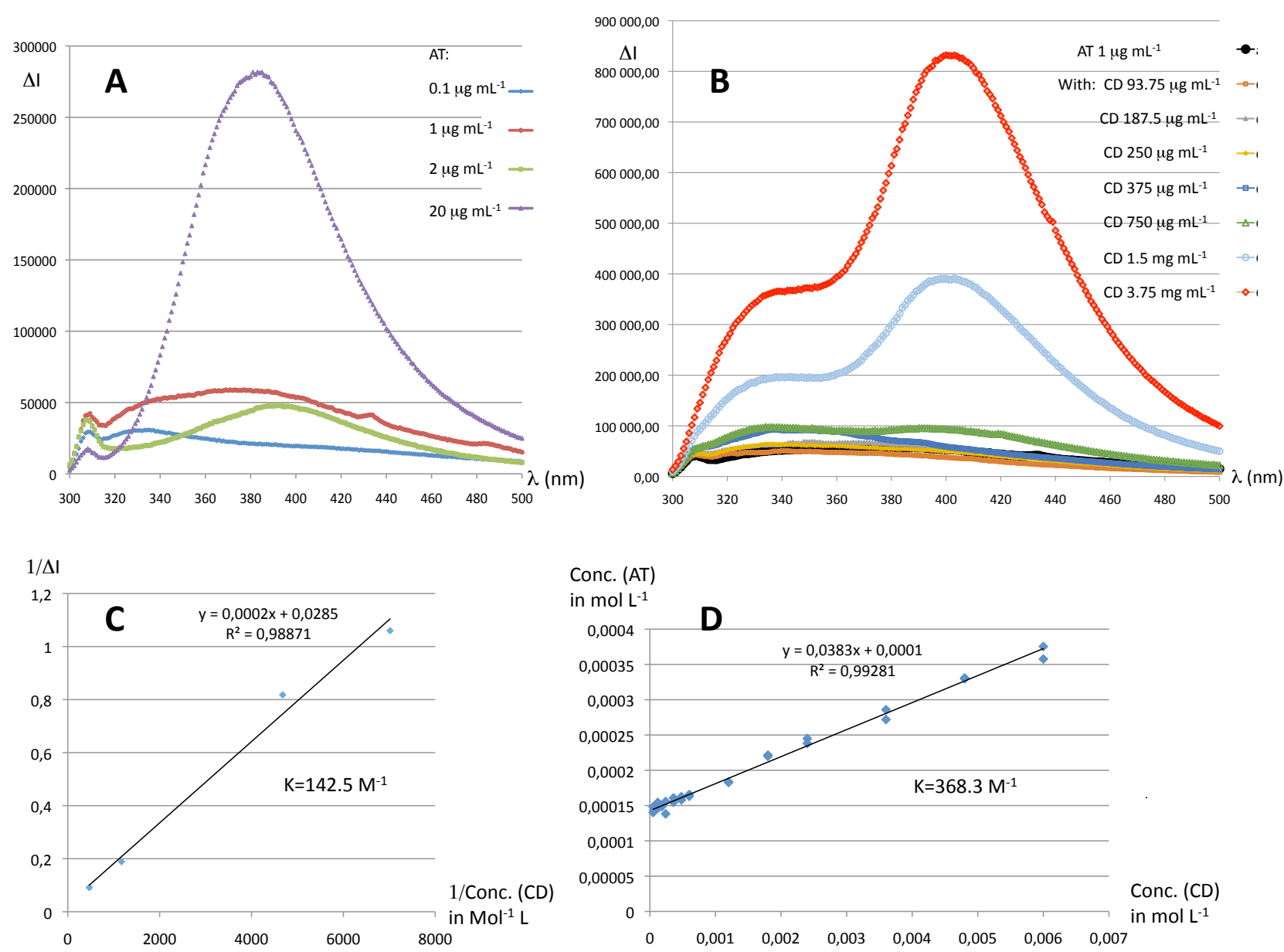

Figure 2: Inclusion complexation of atorvastin by amino- $\beta-C D$ polymer confirmed by fluorescence and UV-vis spectroscopies. (A) Hydrophobic aggregation properties of AT at increasing concentration, leading to an increase in fluorescence intensity together with a red shift in emission wavelength (excitation $276 \mathrm{~nm}$, emission around $381 \mathrm{~nm}$ ); (B) Strong increase in AT fluorescence intensity, induced by the presence of amino- $\beta-C D$ polymer, 
explained by inclusion of AT substituents into the apolar cavities of $\beta$-cyclodextrins; $(\mathbf{C})$ Double reciprocal plot (Benesi-Hildebrand) of $1 / \Delta \mathrm{I}$ versus $1 / \mathrm{C}(\mathrm{CD})$, giving a graphical estimate of 1:1 inclusion complex stability constant (linear plot); (D) Solubilisation measurements, giving a graphical estimate of 1:1 inclusion complex stability constant (linear plot).
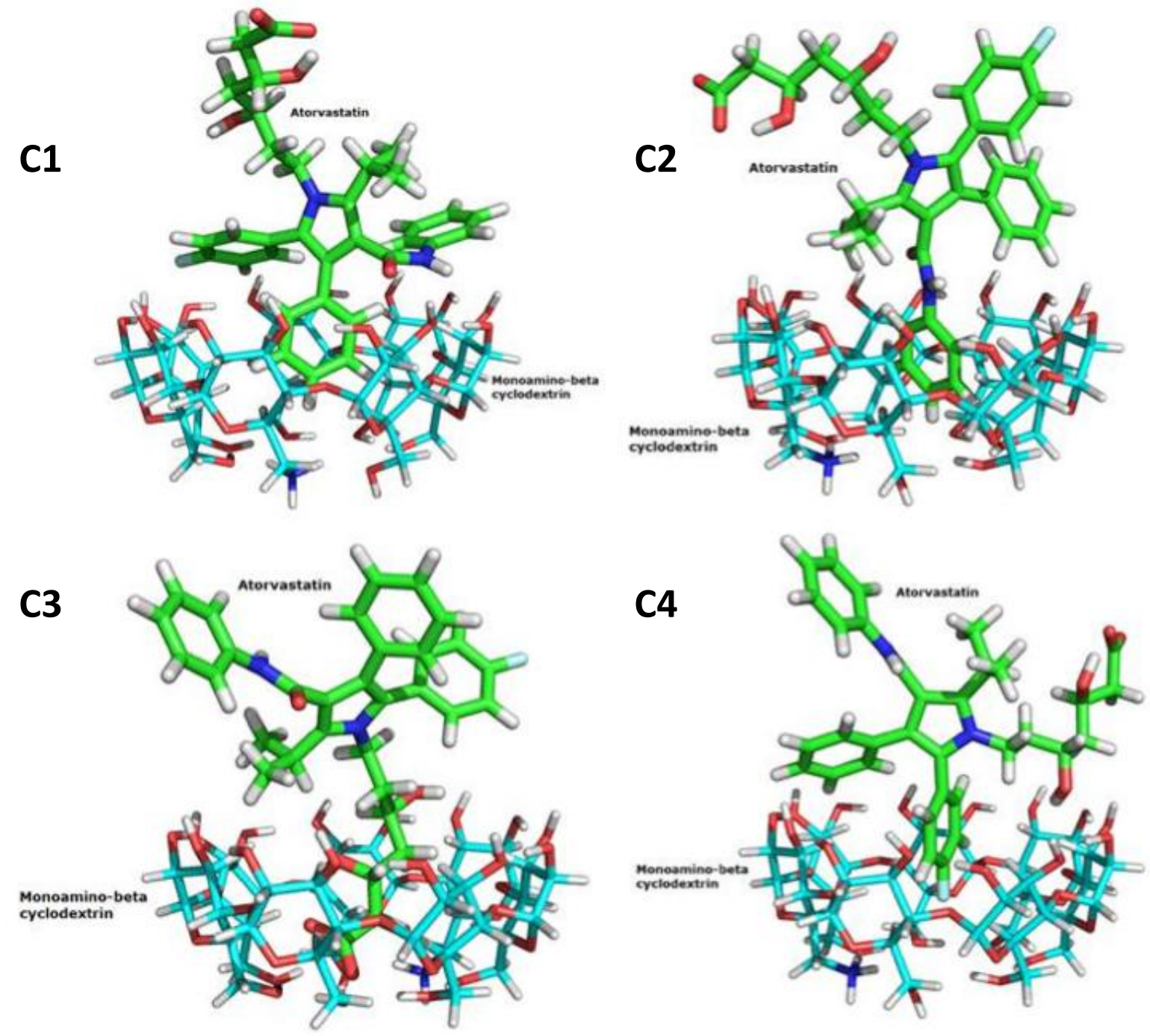

Figure 3: Four potential complexes (C1-C4) of atorvastatin and amino- $\beta-C D$ by molecular dynamics simulations. 

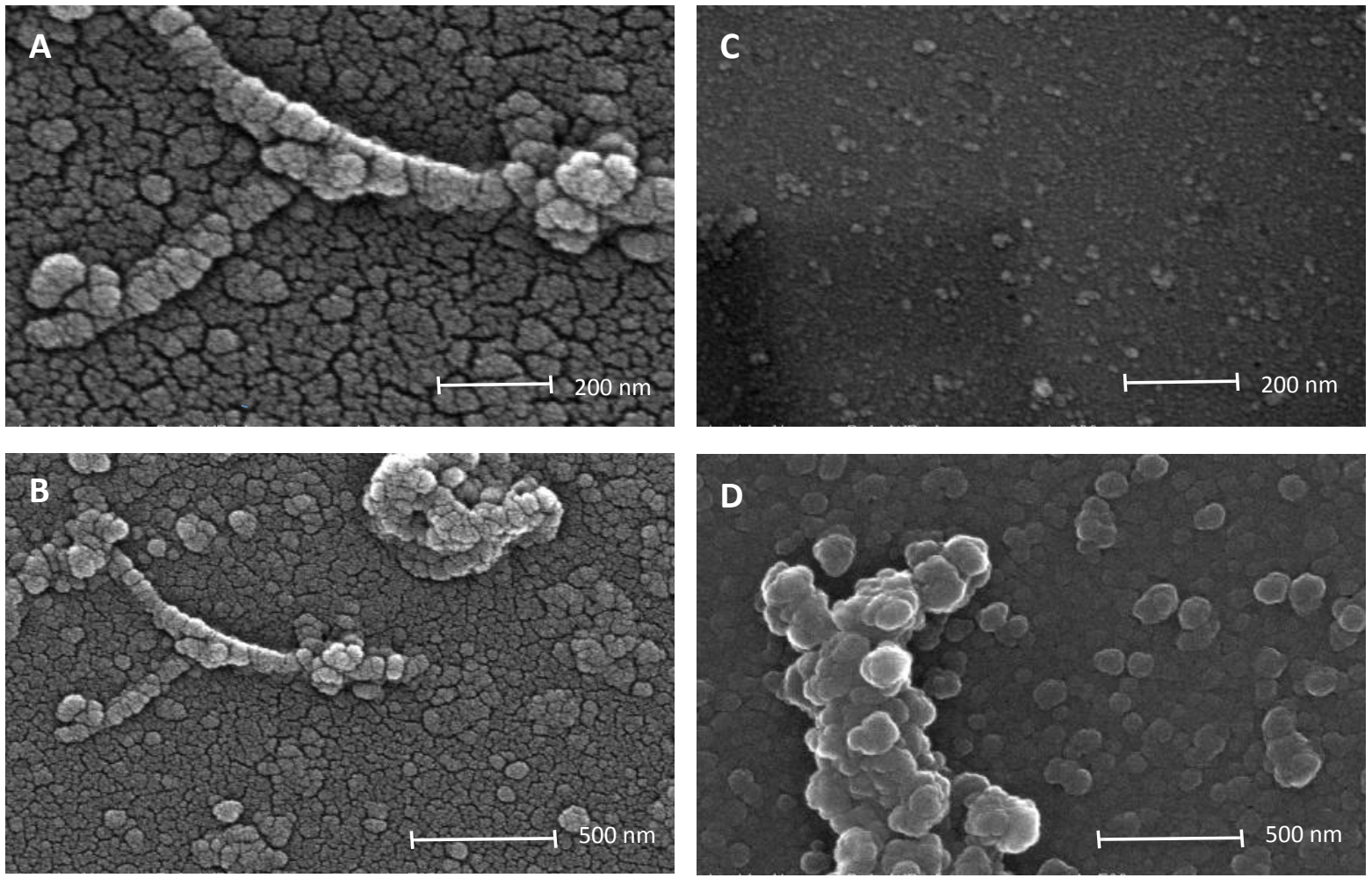

Figure 4: Scanning Electron Micrographs of compounds deposited on glass from aqueous solutions: (A, B) Atorvastatin, (C) Amino- $\beta$-cyclodextrin polymer, (D) Atorvastatin/Amino$\beta$-cyclodextrin polymer Complexes. 

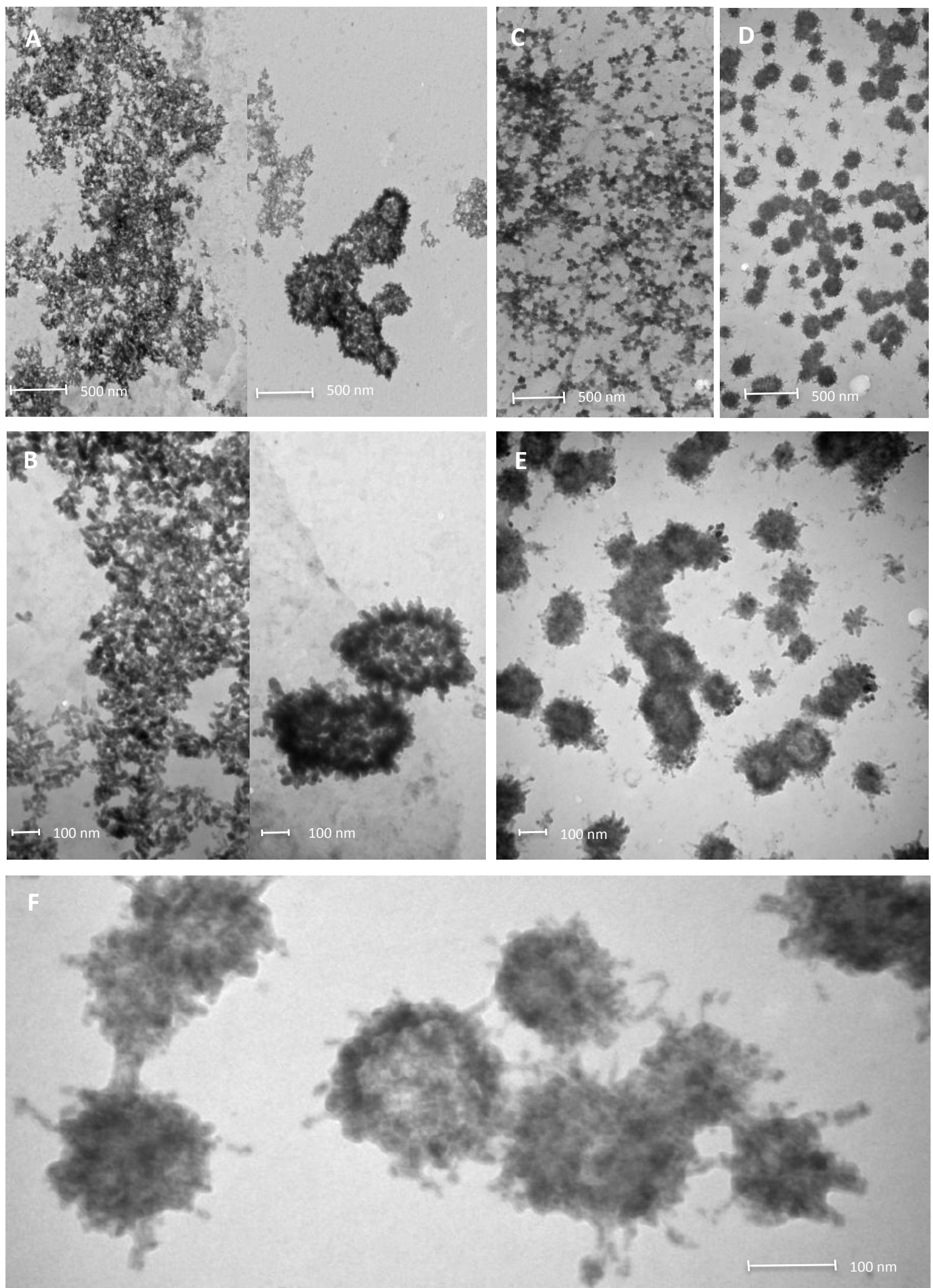

Figure 5: Transmission Electron Micrographs of compounds deposited on carbon coated grids from aqueous solutions: (A, B) Atorvastatin, $(\mathbf{C})$ Amino- $\beta$-cyclodextrin polymer, $(\mathbf{D}, \mathbf{E}$, F) Atorvastatin/Amino- $\beta$-cyclodextrin polymer Complexes. 

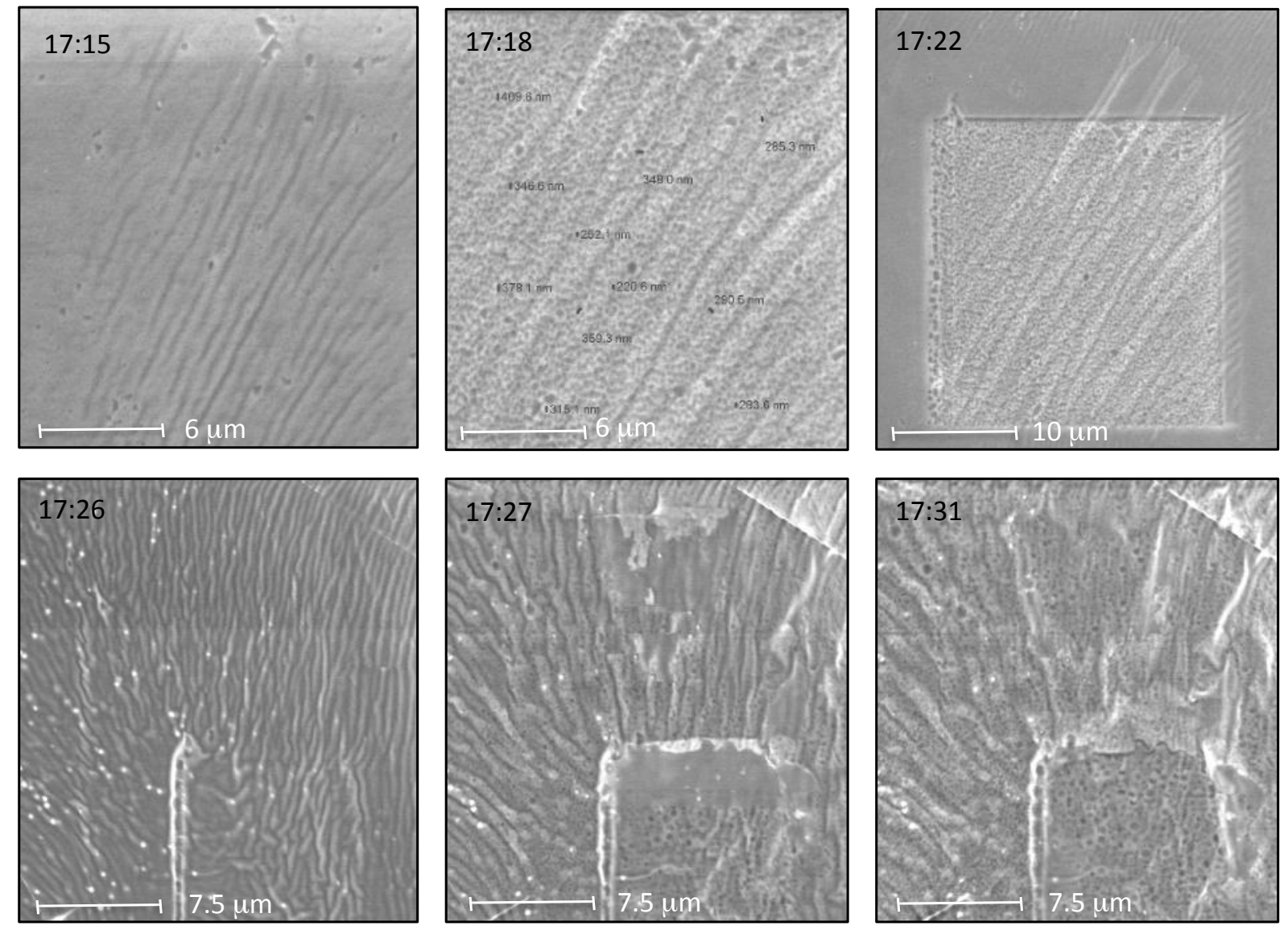

Figure 6: Scanning Electron Micrographs of Amino- $\beta$-cyclodextrin polymer hydrogel: apparition of the porous structure under the electron beam $(5 \mathrm{kV})$ within a few minutes. 

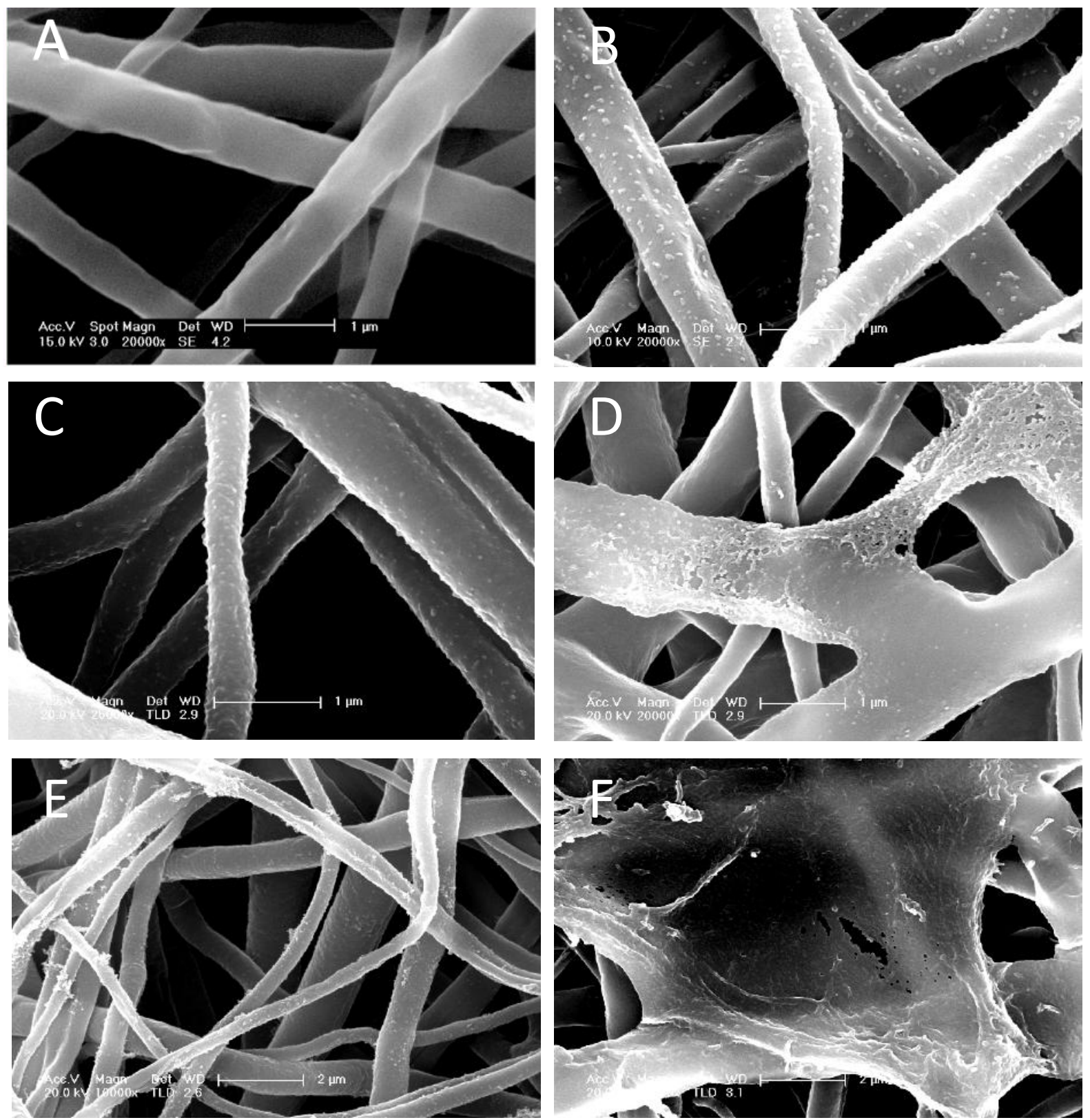

Figure 7: Scanning Electron Micrographs of atorvastatin-functionalised PCL membranes. (A) Non-functionalised control PCL membrane; (B) -PLL-DEX-(CPLX-DEX-)5 "5 cycles" nanoreservoirs of AT-CD complexes (CPLX) and dextran sulfate (DEX), with amino- $\beta$-CD polymer $190 \mu \mathrm{g} \mathrm{mL}{ }^{-1}$; (C) -PLL-DEX-(CD-DEX-) " "5 cycles" nanoreservoirs of amino- $\beta$ CD polymer/dextran sulfate, with CD polymer $1 \mathrm{mg} \mathrm{mL}^{-1}$; (D) -PLL-DEX-(CPLX-DEX-) "5 cycles" nanoreservoirs of AT-CD complexes/dextran sulfate, with CD polymer $1 \mathrm{mg} \mathrm{mL}^{-1}$; (E) -PLL-DEX-(CD-DEX-) $)_{10}$ "10 cycles" nanoreservoirs of CD polymer/dextran sulfate, with CD polymer $1 \mathrm{mg} \mathrm{mL}^{-1}$; (F) -PLL-DEX-(CPLX-DEX-) $)_{10}$ "10 cycles" nanoreservoirs of AT-CD complexes/dextran sulfate, with $\mathrm{CD}$ polymer1 $\mathrm{mg} \mathrm{mL}^{-1}$. 


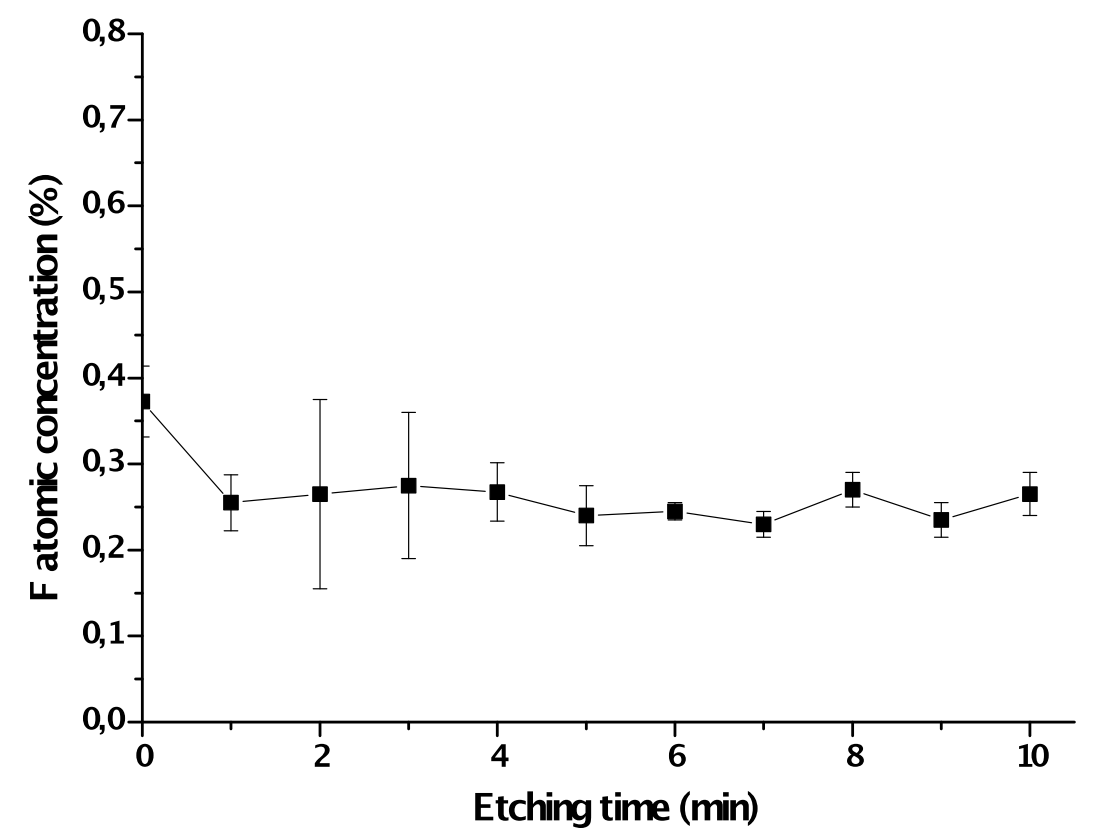

Figure 8: Variation of fluor $(\mathrm{F})$ atomic concentration with etching time obtained from XPS measurements on atorvastatin-functionalised PCL membranes.
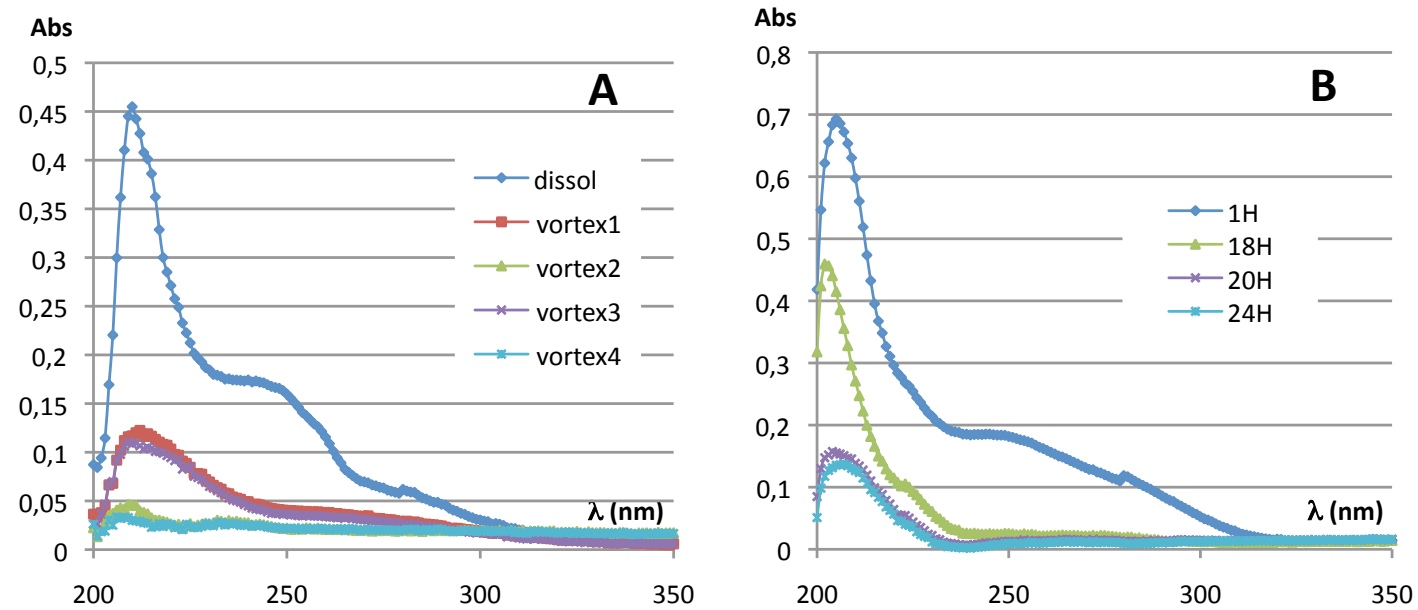

Figure 9: Atorvastatin UV-vis spectra in MES:methanol 1:1 for (A) The recovery of atorvastatin embedded in PCL membrane coating, by successive methanol washings; (B) The follow-up of atorvastatin release with time when changing from MES buffer ( $\mathrm{pH} 5.5$ ) to PBS ( $\mathrm{pH}$ 7.4). 

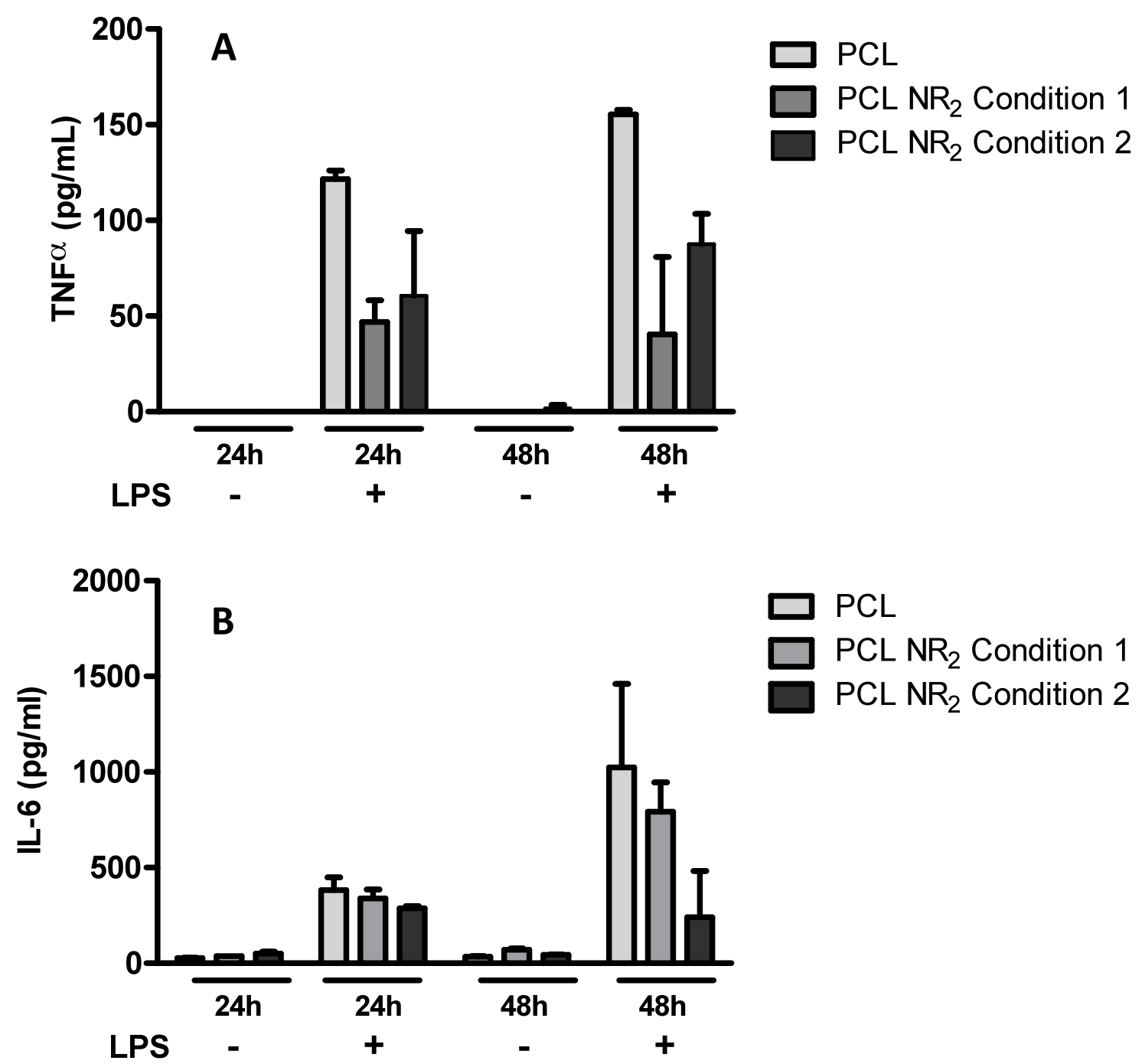

Figure 10: Atorvastatine-coated PCL repress IL-6 and TNF- $\alpha$ production in LPS-activated THP-1 Cells: (A) TNF- $\alpha$ release was determined by ELISA in THP-1 culture supernatants after LPS $(0.1 \mu \mathrm{g} / \mathrm{mL})$ stimulation for 6,24 and $48 \mathrm{~h}$, in the presence of empty (PCL) or atorvastatine-coated PCL membranes (Condition1: AT complexes formed with CD polymer 1 $\mathrm{mg} \mathrm{mL}^{-1}, 2$ cycles nanoreservoirs; Condition 2: AT complexes formed with CD polymer 10 $\mathrm{mg} \mathrm{mL} \mathrm{m}^{-1}, 2$ cycles nanoreservoirs); (B) IL-6 release was determined by ELISA in culture supernatants after stimulation of THP-1 cells in the same conditions as in (A). Quantification was made in duplicates and two independent experiments were realized. Histograms represent mean + -- s.e.m. 